\title{
Postnationale Demokratie - Gibt es ein Menschenrecht auf transnationale Selbstbestimmung?
}

\author{
Thomas Groß*
}
A. Einleitung
B. Demokratie und Kollektiv
I. Die Tradition der nationalstaatlichen Demokratie....
II. Die Pluralisierung des Volksbe- griffs .......................... 130
III. Verfassungsvergleichende Beobach- tungen .......................... 131
1. Bundesrepublik Deutschland.... 131
2. Schweizer Eidgenossenschaft.... 132
3. Vereinigte Staaten von Ame- rika ........................... 133
4. Indien ....................... 133
5. Europäische Union ............. 134
C. Demokratie und Individuum .......... 135
I. Die Kongruenz von Betroffenheit und Mitwirkungsrechten ........... 135
II. Die Diskrepanz zwischen Rechtsau- torschaft und Rechtsbetroffenheit... 136
III. Demokratie als individuelles Recht in Mehrebenensystemen .............. 138

D. Mögliche Formen der Institutionalisierung .............................. 143

I. Die Verbesserung der Mitwirkung nationaler Parlamente.............. 143

II. Die Verbesserung der Transparenz . 144

III. Die Schaffung einer globalen parlamentarischen Versammlung........ 145

IV. Die Schaffung eines Weltparlaments ............................ 146

E. Einwände ........................ 148

I. Der Einwand der territorialen Größe ............................ 148

II. Der Einwand der fehlenden Homogenität .......................... 149

III. Der Einwand des fehlenden Konsenses.............................. 151

IV. Der Einwand der Tyrannisgefahr... 152 F. Ausblick .......................... 153

\section{A. Einleitung}

Während in der Politikwissenschaft wie in der politischen Philosophie seit mehreren Jahrzehnten intensiv über Formen der Demokratie, die jenseits des Nationalstaats liegen, diskutiert wird, ${ }^{1}$ wird in der juristischen Debatte nach wie vor die Möglichkeit der Institutionalisierung demokratischer Entscheidungsverfahren auf globaler Ebene überwiegend ausgeschlossen. ${ }^{2}$ Auch in der deutschen Völkerrechtswissenschaft hat die Frage der Demokratisierung der internationalen Ordnung bisher nur eine geringe Rolle gespielt. ${ }^{3}$ Für viele ist Demokratie nur als Organisationsform eines Volkes in einem Nationalstaat vorstellbar. Lediglich über die Übertragbarkeit von Demokratie

* Prof. Dr. Thomas Groß lehrt Öffentliches Recht, Umweltrecht und Verwaltungswissenschaft an der Universität Frankfurt am Main. Für wertvolle Hinweise gilt Armin v. Bogdandy, Brun-Otto Bryde, Pascale Cancik, Anuscheh Farahat, Günter Frankenberg, Stefan Kadelbach und Anne Peters Dank.

1 Vgl. die prominente Stimme von J. Habermas, Die postnationale Konstellation, Frankfurt am Main 1998; weitere Nachweise bei I. Pernice, Europäisches und nationales Verfassungsrecht, VVDStRL 60 (2001), S. 148 (152), und in den folgenden Fußnoten.

2 Vgl. z.B. U. Volkmann, Setzt Demokratie den Staat voraus?, AöR 127 (2002), S. 575 (598ff.); C. Schönberger, Unionsbürger, Tübingen 2005, S. 157 f.; C. D. Classen, Demokratische Legitimation im offenen Rechtsstaat, Tübingen 2009, S. 115 f.; S. Müller-Franken, Die demokratische Legitimation öffentlicher Gewalt in den Zeiten der Globalisierung, AöR 134 (2009), S. 542 ff.; skeptisch auch R. Poscher, Das Verfassungsrecht vor den Herausforderungen der Globalisierung, VVDStRL 67 (2008), S. 160 (174 ff.).

3 A. $v$. Bodgandy, Demokratie, Globalisierung, Zukunft des Völkerrechts - eine Bestandsaufnahme, ZaöRV 63 (2003), S. 853 (866). 
auf die supranationale Ebene der Europäischen Union gibt es eine intensive Auseinandersetzung. ${ }^{4}$ Für eine demokratische Struktur der EU hat das Bundesverfassungsgericht jedoch im Urteil über den Vertrag von Lissabon hohe Hürden errichtet, die auf einer klaren Priorität nationalstaatlicher Demokratie beruhen. ${ }^{5}$ Der zweite Senat verwendet allein den Begriff der Souveränität mehr als 50-mal, obwohl dieser im Grundgesetz gar nicht vorkommt. ${ }^{6}$ Sicherheitshalber wird die Souveränität der Bundesrepublik als „unaufgebbares Verfassungsgebot" in Art. 79 Abs. 3 GG verankert. ${ }^{7}$ Vielleicht ist aber gerade die intensive Verteidigung des Nationalstaats als Symptom seiner Krise aufzufassen.

Gesellschaftliche Probleme, die an der Leistungsfähigkeit der bestehenden internationalen Ordnung, die auf der souveränen Gleichheit der Staaten beruht (vgl. Art. 2 Nr. 1 UN-Charta), zweifeln lassen, gibt es jedenfalls genug. Ein besonders drängendes Phänomen, dessen globale Reichweite unbestreitbar ist, ist der Klimawandel. Das Phänomen der Erderwärmung verdeutlicht besonders eindringlich, dass territorial begrenzte politische Gemeinschaften das Problem nicht in den Griff bekommen können, sondern internationales Handeln erforderlich ist. ${ }^{8}$ Das Scheitern der Klimakonferenz von Kopenhagen im Dezember 2009 ist ein aktueller Beleg dafür, dass die bestehenden Entscheidungsstrukturen nicht geeignet sind, um solche dringenden Probleme zu bewältigen.

Die ökonomische Interdependenz der Weltwirtschaft ist ebenfalls unbestreitbar. ${ }^{9}$ Sie ist in der globalen Finanzmarktkrise 2008/09 besonders deutlich geworden. Notleidende US-amerikanische Immobilienkredite wurden weltweit gehandelt, die Folgen der Krise für viele Volkswirtschaften waren gravierend. Auf der anderen Seite hat die zunehmende Verflechtung die Kluft zwischen den ärmsten und den reichsten Staaten nicht verringert, sondern vergrößert, aber immerhin geht der Anteil der Weltbevölkerung, der in extremer Armut lebt, leicht zurück. ${ }^{10}$ Nach wie vor ist in

4 Vgl. dazu z.B. W. Kluth, Die demokratische Legitimation der Europäischen Union, Berlin 1995; M. Kaufmann, Europäische Integration und Demokratieprinzip, Baden-Baden 1997; A. Peters, Elemente einer Theorie der Verfassung Europas, Berlin 2001, S. 626 ff.; H. Bauer/P.M. Huber/K.-P. Sommermann, Demokratie in Europa, Tübingen 2005; A. v. Komorowski, Demokratieprinzip und Europäische Union, Berlin 2010.

5 BVerfGE 123, $267 \mathrm{ff}$.

6 E. Röper, Der Souveränitäts- und Volksbegriff des Bundesverfassungsgerichts, DÖV 2010, S. 285 (286); krit. auch J. E. K. Murkens, „We Want Our Identity Back“ - The Revival of National Sovereignty in the German Federal Constitutional Court's Decision on the Lisbon Treaty, Public Law 2010, S. 530 (533 ff.).

7 C. Hillgruber, Der Nationalstaat in der überstaatlichen Verflechtung, in: J. Isensee/P. Kirchhof (Hrsg.), Handbuch des Staatsrechts, Bd. II, 3. Aufl., Heidelberg 2004, $\$ 32$ Rn. $40 \mathrm{f}$.

8 Vgl. A. McGrew, Demokratie ohne Grenzen? Globalisierung und die demokratische Theorie und Politik, in: U. Beck (Hrsg.), Politik der Globalisierung, Frankfurt am Main 1998, S. 374 (382).

9 Vgl. den Überblick bei D. Held, Soziale Demokratie im globalen Zeitalter, Frankfurt am Main 2007, S. $47 \mathrm{ff}$.

10 Auch hierzu mit Zahlen Held, Demokratie (Fn. 9), S. 66 ff. 
vielen Bereichen eine selektive Liberalisierung der Weltmärkte zu beobachten, die auf divergenten Interessenlagen der Staaten und entsprechenden politischen Entscheidungen beruht. Auch hier führt aber das im Völkerrecht geltende Konsensprinzip dazu, dass um gemeinsame Regelungen, etwa im Rahmen der WTO, zum Teil über Jahrzehnte gerungen werden muss.

Dass der Terrorismus ebenfalls ein globales Phänomen ist und seine Bekämpfung einer gemeinsamen Anstrengung bedarf, ist seit den Anschlägen vom 11. September 2001 jedem deutlich geworden. Nicht nur aufgrund der aktuellen Situation in Afghanistan ist es aber sehr fraglich, ob militärische Mittel dabei eine so zentrale Rolle spielen sollten, wie es bisher der Fall war. Zudem ist die Kehrseite, dass es im Rahmen der Terrorismusbekämpfung zu Menschenrechtsverletzungen auch durch westliche Demokratien kam. ${ }^{11}$ Die Missachtung grundlegender Menschenrechte ist aber auch in anderen Kontexten nach wie vor in vielen Ländern der Erde zu beklagen. ${ }^{12}$ Auch mehr als sechzig Jahre nach der Verabschiedung der Allgemeinen Erklärung der Menschenrechte gibt es hier viel Handlungsbedarf.

Dass die zunehmende internationale Interdependenz eine globale institutionelle Ordnung erforderlich macht und dass das alte westfälische System mit seiner starken Betonung staatlicher Souveränität nicht mehr geeignet ist, Frieden und Wohlstand zu sichern, ist eine Erkenntnis, die sich schon nach dem ersten Weltkrieg allmählich durchsetzte. Deshalb entstand eine Vielzahl von internationalen Organisationen, sowohl auf der regionalen Ebene, insbesondere in Europa, wie auch im globalen Rahmen, wo die Vereinten Nationen und die ihr zugeordneten Organisationen in vielfältiger Weise Einfluss auf nationale Entscheidungen nehmen. Von ihnen wird inzwischen praktisch jedes Politikfeld erfasst. ${ }^{13}$ Die äußere Souveränität der Einzelstaaten kann die Sicherheit der eigenen Bevölkerung, verstanden in einem umfassenden Sinn, nicht mehr schützen und erfordert deshalb die Fähigkeit zur Kooperation. ${ }^{14}$

Allerdings sind die internationalen Organisationen von den demokratischen Prozessen wie von den öffentlichen Meinungsforen, die nach wie vor überwiegend im einzelstaatlichen Rahmen institutionalisiert sind, weit entfernt. Die Verhandlungen

11 Vgl. zum amerikanischen „Folterdiskurs“ G. Frankenberg, Staatstechnik, Frankfurt am Main 2010, S. $277 \mathrm{ff}$.

12 Vgl. amnesty international, Report 2010, Frankfurt am Main 2010.

13 H. Steiger, Brauchen wir eine Weltrepublik?, Der Staat 42 (2003), S. 249 (260); B.-O. Bryde, International Democratic Constitutionalism, in: R. Macdonald/D. Johnston (Hrsg.), Towards World Constitutionalism, The Hague: Nijhoff 2005, S. 103 ff.; Held, Demokratie (Fn. 9), S. 124 ff.; ausführlich nun die Beiträge in A. v. Bogdandy/R. Wolfrum/J. v. Bernstorff/P. Dann/M. Goldmann (Hrsg.), The Exercise of Public Authority by International Institutions, Berlin u.a. 2010.

14 D. Held, Democracy and the Global Order, Stanford: Stanford University Press 1995, S. 87 f.; J. Habermas, Eine politische Verfassung für die pluralistische Weltgesellschaft?, in: ders., Zwischen Naturalismus und Religion, Frankfurt am Main 2005, S. 324 (332). 
werden von Vertretern der Exekutiven der Mitgliedstaaten geführt, häufig finden sie hinter verschlossenen Türen statt. Auch wenn die nationalen Parlamente völkerrechtlichen Verträgen zustimmen müssen, sind sie durch die von den Regierungen ausgehandelten Ergebnisse präjudiziert. ${ }^{15}$ Auf der anderen Seite ist die Struktur des für den Schutz von Frieden und Menschenrechte zentralen UN-Sicherheitsrates problematisch, da er durch die Vetorechte der ständigen Mitglieder in der Vergangenheit gerade bei humanitären Katastrophen oft nicht handlungsfähig war. ${ }^{16}$ Diese Sonderrechte der fünf Supermächte sind historisch erklärbar, stehen aber in einem Spannungsverhältnis mit der für das klassische Völkerrecht grundlegenden Gleichheit zwischen den Staaten. ${ }^{17}$

Die miteinander verflochtenen Entwicklungen des faktischen Autonomieverlustes selbst der großen Industrieländer und der in immer mehr Politikfelder ausgreifenden transnationalen Kooperation führen zu einer Erosion der nationalstaatlichen Souveränität von außen. ${ }^{18}$ Die ursprünglich selbstverständliche Annahme einer Kongruenz zwischen politischen Entscheidungsträgern und den Betroffenen im Nationalstaat besteht nicht mehr. ${ }^{19}$ Auch Verteidiger der nationalstaatlichen Demokratie müssen zugeben, dass deshalb der Grundsatz der demokratischen Selbsteinwirkung in wichtigen Bereichen leerläuft. ${ }^{20}$

Ziel dieses Beitrags ist es, die These über den notwendigen Zusammenhang zwischen Nation und Demokratie in Frage zu stellen und als konzeptionelle Alternative eine Demokratietheorie zu skizzieren, die nicht vom Volk als Kollektiv, sondern vom Individuum ausgeht. Dieser Ansatz transzendiert das Selbstverständnis der geltenden Verfassungsordnungen wie auch die westfälische Grundstruktur des Völkerrechts. Dennoch lassen sich für diese Weiterentwicklung der pluralistischen Demokratietheorie ${ }^{21}$ Anknüpfungspunkte sowohl im Verfassungsvergleich föderaler Ordnungen (B.) als auch in der Theorie der Grund- und Menschenrechte (C.) finden. Auf dieser Grundlage werden mögliche Formen der Institutionalisierung globaler Demokratie skizziert (D.) und einige wichtige Einwände diskutiert (E.).

15 K.-P. Sommermann, Demokratie als Herausforderung des Völkerrechts, in: P.-M. Dupuy/B. Fassbender/M. Shaw/K.-P. Sommermann (Hrsg.), Völkerrecht als Wertordnung, Common Values in International Law, FS Tomuschat, Kehl u.a. 2006, S. 1051 (1060).

16 Habermas, Weltgesellschaft (Fn. 14), S. 352 ff.

17 F. Müller, Demokratie zwischen Staatsrecht und Weltrecht, Berlin 2003, S. 132 f.

18 B.-O. Bryde, Die bundesrepublikanische Volksdemokratie als Irrweg der Demokratietheorie, StWStPrax 1994, S. 305 (307); O. Höffe, Demokratie im Zeitalter der Globalisierung, München 1999, S. $166 \mathrm{ff}$.

19 Held, Global Order (Fn. 14), S. 16 ff.

20 I. Maus, Verfassung oder Vertrag. Zur Verrechtlichung globaler Politik, in: P. Niesen/B. Herborth (Hrsg.), Anarchie und kommunikative Freiheit, Frankfurt am Main 2007, S. 350 (373).

21 T. Groß, Das Kollegialprinzip in der Verwaltungsorganisation, Tübingen 1999, S. 163 ff.; A. Hanebeck, Der demokratische Bundesstaat des Grundgesetzes, Berlin 2004, S. 84 ff.; H.-H. Trute, Die demokratische Legitimation der Verwaltung, in: W. Hoffmann-Riem/E. Schmidt-Aßmann/A. Voßkuhle (Hrsg.), Grundlagen des Verwaltungsrechts, Bd. 1, München 2006, \6 Rn. 17 ff. 


\section{B. Demokratie und Kollektiv}

Demokratie ist ein kollektives Recht, das einem Volk zusteht, das historisch entstanden ist und sich durch den Prozess der Verfassungsgebung als Subjekt eines Staates konstituiert. Diese Prämisse ist für das traditionelle nationalstaatsbezogene Demokratieverständnis prägend (I.). In föderalen Ordnungen erfolgt jedoch bereits eine Pluralisierung des Volksbegriffs (II.). Sie kann durch verfassungsvergleichende Beobachtungen belegt werden (III.).

\section{Die Tradition der nationalstaatlichen Demokratie}

Dass die territoriale Organisation liberaler Demokratie mit der souveränen Staatlichkeit deckungsgleich ist, ist in der Geschichte des westlichen Staatsdenkens tief verwurzelt. ${ }^{22}$ In der Tradition der nationalstaatlichen Demokratie gilt das Volk als Träger des Staates und als Autor der Verfassung, wie es sich etwa in der Präambel des Grundgesetzes und in Art. 20 Abs. 2 GG niedergeschlagen hat. Das Volk wird dabei vom Bundesverfassungsgericht als Gesamtheit der deutschen Staatsangehörigen und der nach Art. 116 Abs. 1 GG gleichgestellten Personen definiert. ${ }^{23}$ Art. 3 der französischen Verfassung formuliert explizit, dass die nationale Souveränität beim Volk liegt. Auch dort steht das Wahlrecht für das Parlament nur den Staatsangehörigen zu. ${ }^{24}$ Art. 4 Abs. 1 der polnischen Verfassung lautet ähnlich: „Die oberste Gewalt der Republik Polen steht der Nation zu. " Das Recht zur Teilnahme an Wahlen und Abstimmungen wird in Art. 62 Abs. 1 ausdrücklich auf polnische Staatsbürger beschränkt.

Als Faktoren, durch die sich das Volk definiert, werden insbesondere Geschichte, Sprache, Kultur, Religion genannt. ${ }^{25}$ Oft werden demos als Gesamtheit der Bürger und ethnos als historische Schicksalsgemeinschaft identifiziert. ${ }^{26}$ Dies zeigt sich etwa an der Formulierung des Bundesverfassungsgerichts im Maastricht-Urteil, das Volk

22 McGrew, Grenzen (Fn. 8), S. 381.

23 BVerfGE 83, 37 (51).

24 P. Ardant/B. Mathieu, Institutions politiques et droit constitutionnel, 20. Aufl., Paris: L.G.D.J. 2008, S. 188, zur größeren Offenheit der Revolutionsverfassungen vgl. R. Keil, Kants Demokratieverständnis und Ausländerwahlrechte heute, Baden-Baden 2005, S. $412 \mathrm{ff}$.

25 P. Kirchhof, Der Staat als Organisationsform politischer Herrschaft und rechtlicher Bindung, DVBl. 1999, S. 637 (643); Müller-Franken, Legitimation (Fn. 2), S. 565.

26 So z.B. J. Isensee, Staat und Verfassung, in: ders./P. Kirchhof (Hrsg.), Handbuch des Staatsrechts, Bd. 1, 1. Aufl., Heidelberg 1987, $\$ 13$ Rn. 112 f.; E.-W. Böckenförde, Demokratie als Verfassungsprinzip, in: J. Isensee/P. Kirchhof (Hrsg.), Handbuch des Staatsrechts, Bd. 1, 1. Aufl., Heidelberg 1987, $\$ 22$ Rn. 26; zur Unterscheidung vgl. S. Benhabib, Kosmopolitismus und Demokratie, Frankfurt am Main 2008, S. 43 (65 f.); zur Kritik grundlegend F. Hanschmann, Der Begriff der Homogenität in der Verfassungslehre und Europarechtswissenschaft, Heidelberg u.a. 2008, S. $41 \mathrm{ff}$. 
sei „relativ homogen“. ${ }^{27}$ Dementsprechend findet man auch die These, wonach es keine Demokratie ohne Volk in diesem Sinn geben könne. ${ }^{28}$

Eine weitere Schlussfolgerung ist die Gleichsetzung von Volks- und Staatssouveränität. ${ }^{29}$ Wenn das durch die Staatsangehörigkeit definierte Volk die höchste Quelle der Staatsgewalt ist, darf es auch keine übergeordnete Organisation geben, deren Entscheidungen für die nationalen Instanzen verbindlich sind. Aus dieser Perspektive muss die Integration in eine internationale Gemeinschaft auf einer verfassungsrechtlichen Ermächtigung beruhen, die gewährleistet, dass nur begrenzte Kompetenzen übertragen werden und kein supranationaler Staat entsteht. ${ }^{30}$

\section{Die Pluralisierung des Volksbegriffs}

Die Fixierung der Demokratie auf ein ethnisch homogenes Volk in Teilen der deutschen Staatsrechtslehre ist unhistorisch, unrealistisch und für große Teile der Welt untauglich. Der Nationalstaat westeuropäischer Prägung ist nur ein historischer Grenzfall einer großen Vielfalt bündischer und imperialer Staatsformen. ${ }^{31}$ Selbst der Prototyp des Nationalstaates, die französische Republik, ist in jahrhundertelangen Auseinandersetzungen aus einer großen Vielfalt von Territorien entstanden, deren insbesondere sprachliche Homogenisierung erst nach der Revolution von 1789 erzwungen wurde. Auch die deutsche Geschichte des 19. Jahrhunderts belegt, dass kollektive Identitäten eher gemacht als vorgefunden werden. ${ }^{32}$ Deshalb ist die Aussage richtig: „Völker fallen nicht vom Himmel, sie entwickeln sich im Lauf der Geschichte". 33

Nation und Volk müssen folglich unterschieden werden. ${ }^{34}$ In den meisten Staaten der Welt, auch in stabilen Demokratien, herrscht bei zwei der genannten Faktoren, Sprache und Religion, keine Homogenität. Insbesondere die gewaltsamen Ausein-

27 BVerfGE 89, 155 (186). Im Lissabon-Urteil fehlt dieser Begriff, vgl. G. Britz, Vom kulturellen Vorbehalt zum Kulturvorbehalt in der bundesverfassungsgerichtlichen Demokratietheorie des LissabonUrteils?, Europarecht - Beiheft 1/2010, S. 151 (164 ff.).

28 Z.B. J. Neyer, Justice or Democracy, in: R. Nickel (Hrsg.), Conflict of Laws and Laws of Conflict in Europe and Beyond, Oslo: ARENA 2009, S. 41 (43).

29 So z.B. U. Di Fabio, Der neue Art. 23 des Grundgesetzes, Der Staat 32 (1993), S. 191 (205 f.); C. Seiler, Der souveräne Verfassungsstaat zwischen demokratischer Rückbindung und überstaatlicher Einbindung, Tübingen 2005, S. 132 ff.; implizit auch BVerfGE 123, 267 (340 ff.); kritisch z.B. Volkmann, Demokratie (Fn. 2), S. 585.

30 BVerfGE 89, 155 (187f.); 123, 267 (347 ff.).

31 H. Brunkhorst, There Will Be Blood, in: ders. (Hrsg.), Demokratie in der Weltgesellschaft, Soziale Welt, Sonderband 18, Nomos 2009, S. 99 (106).

32 J. Habermas, Was ist ein Volk? Zum politischen Selbstverständnis der Geisteswissenschaften im Vormärz, in: ders., Postnationale Konstellation (Fn. 1), S. 37.

33 S. Benhabib, Die Rechte der Anderen, Frankfurt am Main 2008, S. 84; ähnlich R. Knieper, Nationale Souveränität, Frankfurt am Main 1991, S. 172; M. Kotzur, Demokratie als Wettbewerbsordnung, VVDStRL 69 (2010), S. 173 (198).

34 I. Maus, Zur Aufklärung der Demokratietheorie, Frankfurt am Main 1992, S. 203 ff.; Benhabib, Kosmopolitismus (Fn. 26), S. 129 (150); zur Diskussion auch H. Abromeit, Wozu braucht man Demokratie?, Opladen 2002, S. 115 ff. 
andersetzungen beim Zerfall der Bundesrepublik Jugoslawien haben deutlich gemacht, welches Konfliktpotential besteht, wenn eine historisch entstandene Vielfalt nicht respektiert wird. Umgekehrt heißt das aber auch, dass ein Volk durch gemeinsame politische Praxis entstehen kann. ${ }^{35}$

Eine in vielen Varianten verbreitete Methode zum Umgang mit innerer Heterogenität ist der Föderalismus. In bündischen Systemen lässt sich eine Pluralisierung des Volksbegriffs feststellen, die gerade anerkennt, dass das Volk nicht homogen ist. Wenn die Staatsgewalt auf mehrere Ebenen verteilt wird, geraten monistische Konzepte von Volkssouveränität in Erklärungsschwierigkeiten. ${ }^{36}$ Die demokratische Legitimation stützt sich hier auf verschiedene Kollektive mit gestufter Zugehörigkeit.

\section{Verfassungsvergleichende Beobachtungen}

Die Untauglichkeit eines monistischen Konzeptes der Volkssouveränität lässt sich anhand von verschiedenen föderalen Ordnungen belegen. Als Beispiele werden im Folgenden neben Deutschland die Schweiz, die USA, Indien und die Europäische Union aufgeführt. Für alle fünf Verfassungssysteme können in diesem Rahmen nur einige wenige Hinweise zu den einschlägigen Vorschriften in den Verfassungstexten gegeben werden. Die jeweiligen Kontroversen über den Volksbegriff werden dabei nur angedeutet.

\section{Bundesrepublik Deutschland}

Das Grundgesetz verwendet den Begriff des Volkes in verschiedenen Kontexten. Die Präambel und Art. 1 Abs. 2 GG berufen sich auf das „Deutsche Volk“ im Singular. In Art. 28 Abs. 1 S. 2 GG wird dagegen der Terminus „Volksvertretung “ für die drei subnationalen Ebenen Land, Kreis, Gemeinde verwendet. Damit wird das Repräsentationsprinzip auf die territorialen Untereinheiten ausgedehnt und die demokratische Qualität des Föderalismus und der kommunalen Selbstverwaltung betont. ${ }^{37}$ Obwohl diese natürlich nur jeweils die Teile der Wahlberechtigten umfassen, die auf dem Gebiet der jeweiligen Einheit ihren Wohnsitz haben, werden sie im Verfassungstext als „Volk“ bezeichnet. Hierfür passt aber die ethnische Definition des Volkes nicht. Oder stellen auch die Bewohner Hessens, des Main-Taunus-Kreises oder der Gemeinde Schwalmstadt eine „Schicksalsgemeinschaft“ dar? Nach Auffassung des Bundesverfassungsgerichts ist der Begriff des Volkes in Art. 28 Abs. 1 S. 2 GG aber wie in Art. 20 Abs. 2 GG durch die deutsche Staatsangehörigkeit definiert. ${ }^{38}$

35 Maus, Aufklärung (Fn. 34), S. 203 ff.; Bryde, Volksdemokratie (Fn. 18), S. 309 f.

36 Vgl. S. Oeter, Integration und Subsidiarität im deutschen Bundesstaatsrecht, Tübingen 1998, S. 381 ff.; Groß, Kollegialprinzip (Fn. 21), S. 167 f.

37 Bryde, Volksdemokratie (Fn. 18), S. $318 \mathrm{f}$.

38 BVerfGE 83, 37 (50 ff.); zur Diskussion über die Existenz von Landesvölkern ausführlich Hanebeck, Bundesstaat (Fn. 21), S. $102 \mathrm{ff}$. 
Diese strikte Auslegung der Homogenitätsklausel im Hinblick auf den Volksbegriff macht es schwer erklärbar, wieso auf kommunaler Ebene eine Erweiterung der Wahlberechtigung auf Unionsbürger durch die Ergänzung von Art. 28 Abs. 1 S. 3 GG zulässig war. Die Unionsbürger werden dadurch nicht Teil des deutschen Volkes. ${ }^{39}$ Dann ist allerdings schwer verständlich, warum diese Änderung nicht in an Art. 79 Abs. 3 GG scheitert. ${ }^{40}$ Das Demokratieprinzip zählt zu den von dieser Vorschrift geschützten Grundsätzen. Offensichtlich nimmt das Bundesverfassungsgericht, das eine Öffnung der Wahlberechtigung durch Verfassungsänderung im Urteil zum kommunalen Ausländerwahlrecht bereits als möglich benannte, ${ }^{41}$ die Beschränkung der Wahlberechtigung auf Deutsche aus dem Garantiegehalt der Ewigkeitsklausel aus. ${ }^{42}$ Dann kann aber auch eine Ausweitung des Kommunalwahlrechts auf Drittstaatsangehörige aus Staaten außerhalb der EU nicht unter die Sperrwirkung von Art. 79 Abs. 3 GG fallen. ${ }^{43}$ Der Volksbegriff des Grundgesetzes ist also keineswegs eindeutig.

\section{Schweizer Eidgenossenschaft}

Die Präambel der Schweizer Bundesverfassung benennt als Verfassungsgeber „Das Schweizervolk und die Kantone". Die gleiche Formulierung findet sich in Art. 1, wonach das Schweizervolk und die namentlich aufgezählten 26 Kantone die Schweizer Eidgenossenschaft bilden. Dies kann als Ausdruck einer gemeinsamen Verfassungssouveränität mit einer unitarischen und einer föderativen Komponente betrachtet werden. ${ }^{44}$ Die frühere Formulierung mit dem Bezug auf die „Völkerschaften der 23 souveränen Kantone“ wurde im Rahmen der Gesamtrevision der Verfassung im Jahr 1999 gestrichen. ${ }^{45}$ Andererseits wird betont, dass das Schweizervolk wegen der sprachlichen und religiösen Vielfalt keine „natürliche Einheit“ bilde, sondern nur durch die Geschichte verbunden sei. ${ }^{46}$

Auch die Präambeln vieler Kantonsverfassungen verwenden den Begriff „Volk“. So beginnt etwa die Präambel der Zürcher Kantonsverfassung von 2005 mit der For-

39 Classen, Legitimation (Fn. 2), S. 35.

40 Vgl. z.B. U. Volkmann, in: K.H. Friauf/W. Höfling (Hrsg.), GG, Berlin, Loseblatt (Stand 2010), Art. 20 (3. Teil), Rn. 30 Fn. 160; weitere Nachweise bei F. Hanschmann, „Die Ewigkeit dauert lange, besonders gegen Ende“ - eine rechtliche (Neu)Bewertung des kommunalen Wahlrechts für Drittstaatsangehörige, ZParl 2009, S. 74 (76, Fn. 9).

41 BVerfGE 83, 37 (59).

42 Ebenso H. Dreier, in: ders. (Hrsg.), GG, Kommentar, Bd. 2, 2. Aufl., Tübingen 2006, Art. 79 III Rn. 43 m.w.N.

43 Hanschmann, Wahlrecht (Fn. 40), S. 80; sehr ausführlich Keil, Ausländerwahlrechte (Fn. 24), S. 267 ff.; vgl. auch die rechtvergleichenden Hinweise bei Schönberger, Unionsbürger (Fn. 2), S. 435 f.

44 So A. Weber, Europäische Verfassungsvergleichung, München 2010, S. 44.

45 Vgl. F. Hafner/R.J. Schweizer, in: B. Ehrenzeller/P. Mastronardi/R.J. Schweizer/K. Vallender (Hrsg.), Die schweizerische Bundesverfassung, Zürich: Dike 2002, Art. 1 Rn. 5.

46 Hafner/Schweizer (Fn. 45), Art. 1 Rn. 4, ähnlich G. Biaggini, Bundesverfassung der Schweizerischen Eidgenossenschaft, Zürich: Schulthess 2007, Art. 1 Rn. 11; R. Rhinow/M. Schefer, Schweizerisches Verfassungsrecht, 2. Aufl., Basel: Helbing Lichtenhahn 2009, Rn. 252. 
mulierung „Wir, das Volk des Kantons Zürich“. Wie in Deutschland wird also der Volksbegriff nicht allein der zentralen Ebene vorbehalten, sondern auch auf die Teilstaaten angewendet. Es wird sogar die Auffassung vertreten, das Schweizervolk setze sich aus einer „Aufrechnung der kantonalen Völker“ zusammen. ${ }^{47}$

\section{Vereinigte Staaten von Amerika}

„We the People of the United States“ lautet seit ihrer Verabschiedung im Jahr 1787 die Eingangsformel der US-Verfassung. Das englische Wort „people“ ist auch ein Plural. ${ }^{48}$ Die amerikanische Vorstellung des Volkssouveräns ist deshalb nicht durch einen Einheitswillen gekennzeichnet, sondern versteht ihn als ein plurales Volk von Gleichen. ${ }^{49}$

Trotz ihrer Entstehung aus einem Staatenbund findet sich in der Verfassung von 1787 keine Referenz auf die Einzelstaaten. Die wortgleiche Anrufung des Volkes findet sich aber auch in den Verfassungen vieler Einzelstaaten, z.B. in der Präambel der Verfassung von Massachusetts ${ }^{50}$ oder Wisconsin. ${ }^{51}$ Dementsprechend wird auch schon in den Federalist Papers der Volksbegriff sowohl auf die Bundesebene wie auf die Einzelstaaten angewendet. ${ }^{52}$ Das Verhältnis zwischen dem Volk des Bundes und der Staaten ist allerdings umstritten und war bereits Gegenstand mehrerer kontroverser Urteile des Supreme Court. ${ }^{53}$

\section{Indien}

Indien als Vielvölkerstaat mit 22 anerkannten Sprachen ist die größte Demokratie der Welt. ${ }^{54}$ Die Präambel der Verfassung, die im Januar 1950 in Kraft trat, benennt ihren Autor als „We the People of India“. Die spezifische föderale Konstruktion Indiens geht von einer einheitlichen Staatsangehörigkeit aus. ${ }^{55}$ Die Einzelstaaten haben keine autonomen Verfassungen, ihre Staatsorganisation wird vielmehr von der indischen Verfassung selbst festgelegt. Der Zentralstaat hat auch eine umfassende Kompetenz zur Neugliederung der föderalen Einheiten, die v.a. nach sprachlichen Gesichtspunkten erfolgt. ${ }^{56}$

47 Rhinow/Schefer, Verfassungsrecht (Fn. 46), Rn. 254.

48 Darauf weist Bryde, Volksdemokratie (Fn. 18), S. 312, hin; s.a. Hanebeck, Bundesstaat (Fn. 21 ), S. 90.

49 U. Rödel/G. Frankenberg/H. Dubiel, Die demokratische Frage, Frankfurt am Main 1989, S. 58.

50 http://www.mass.gov/legis/const.htm.

51 http://www.legis.state.wi.us/statutes/wisconst.pdf.

52 A. Hamilton/J. Madison/J. Jay, The Federalist Papers, New York u.a.: Bantham Books 1982 (1787/1788), Nr. 46: „The Fœderal and State Governments are in fact but different agents and trustees of the people...".

53 Dazu Hanebeck, Bundesstaat (Fn. 21), S. 87 ff.

54 C. Jürgenmeyer, Die indische Demokratie - eine „funktionierende Anarchie“, in: M. v. Hauff (Hrsg.), Indien - Herausforderungen und Perspektiven, Marburg 2009, S. 73 (73 f.).

55 M. P. Singh/S. Deva, The Constitution of India: Symbol of Unity in Diversity, JöR 53 (2005), S. 649 (678).

56 D. Conrad, Rechtssystem und Verfassung, in: D. Rothermund (Hrsg.), Indien: Kultur, Geschichte, Politik, Wirtschaft, Umwelt - Ein Handbuch, München 1995, S. 409 (418). 
Die indische Verfassung legt großen Wert auf den Schutz von Minderheiten, u.a. durch Vorschriften über die Repräsentation bestimmter Kasten und Stämme in den Parlamenten auf nationaler Ebene und in den Einzelstaaten. ${ }^{57}$ Ihre Wahl erfolgt jedoch durch die allgemeine Wählerschaft. ${ }^{58}$ Die sozialistische Hoffnung auf größere nationale Homogenität hat sich nicht durchgesetzt, heute wird stärker die Vielfalt betont. ${ }^{59}$ Die demokratische Legitimation wird gerade durch die Präsenz vieler kleiner Parteien als stark bewertet. ${ }^{60}$ Dadurch entstand ein Zwang zu konsensorientierter Politik, in der die Fähigkeit zur Argumentation eine große Bedeutung hat. ${ }^{61}$ Trotz der großen sozialen Verwerfungen hat sich Indien in sechs Jahrzehnten als stabile Demokratie erwiesen. ${ }^{62}$

\section{Europäische Union}

Die europäische Integration war ursprünglich eindeutig als „Gemeinschaft unter Völkern“ konzipiert, wie es z.B. in der Präambel zum Vertrag über die Gründung der Europäischen Gemeinschaft für Kohle und Stahl vom 18. April 1951 hieß. Auch die Präambel des EUV in Fassung des Lissabonner Vertrags spricht nach wie vor davon, dass die vertragsschließenden Staatsoberhäupter „die Solidarität zwischen ihren Völkern“ stärken wollen. Ebenso benennt Art. 1 Abs. 2 EUV das Ziel „einer immer engeren Union der Völker Europas“. Eine weitgehend identische Formulierung, die ebenfalls von „Völkern“ im Plural spricht, findet sich in der Präambel des AEUV.

Bemerkenswert ist aber, dass die entsprechende Aussage in Art. 189 EGV, wonach das Europäische Parlament aus „Vertretern der Völker der in der Gemeinschaft zusammengeschlossenen Staaten " durch den Vertrag von Lissabon signifikant geändert wurde. Nach dem neuen Wortlaut ist das EP aus „Vertretern der Unionsbürgerinnen und Unionsbürger" zusammengesetzt (Art. 14 Abs. 2 EUV n.F.). Auch Art. 10 Abs. 2 UAbs. 1 EUV spricht nunmehr davon, dass die „Bürgerinnen und Bürger“ auf Unionsebene unmittelbar im Europäischen Parlament vertreten sind. Damit wird die Konsequenz daraus gezogen, dass seit der Einführung der Unionsbürgerschaft im Vertrag von Maastricht das Wahlrecht zum Europäischen Parlament nur noch am Wohnort anknüpft, so dass seine Abgeordneten nicht Vertreter von durch Staatsan-

57 Singh/Deva, India (Fn. 55), S. 685; zur Diskussion in der verfassunggebenden Versammlung vgl. S. Sen, The Constitution of India, New Delhi u.a.: Oxford University Press 2007, S. 103 ff.

58 Conrad, Rechtssystem (Fn. 56), S. 419.

59 H. Goerlich, Verfassung, Recht und Staat im Wandel Indiens seit 1947, in: v. Hauff (Hrsg.), Indien (Fn. 54), S. 31 (37 ff.)

60 Goerlich, Verfassung (Fn. 59), S. 65 f.

61 Jürgenmeyer, Demokratie (Fn. 54), S. $81 \mathrm{ff}$.

62 Dazu auch N. Glazer, Democracy and Deep Divides, Journal of Democracy 2/21 (2010), S. 5 ff. 
gehörigkeit definierten Völkern, sondern der Unionsbürger sind. ${ }^{63}$ Damit löst sich das Repräsentationsorgan der EU auch konzeptionell von der Vermittlung durch die Nationalstaaten.

\section{Demokratie und Individuum}

Wenn man die Demokratietheorie von der Fixierung auf ein historisch vorgegebenes Volk löst, stellt sich die Frage, wie ihre personelle Basis der demokratischen Selbstbestimmung anders begründet werden kann. Überlappende Zugehörigkeiten zu mehreren Kollektiven lassen sich nur erklären, wenn man die Legitimation auf das einzelne Individuum zurückführt, das im Mittelpunkt jeder Staatstheorie steht, die sich der Tradition der Aufklärung verbunden fühlt. Ausgangspunkt ist die Philosophie von Immanuel Kant (I.). Auf dieser konzeptionellen Grundlage wird deutlich, dass die heutigen Demokratien durch eine Diskrepanz zwischen Mitwirkungsrechten und Betroffenheit charakterisiert sind (II.). Diese Diskrepanz kann überwunden werden, wenn man Demokratie konsequent als individuelles Recht versteht und dadurch mit Mehrebenensystemen kompatibel macht (III.).

\section{Die Kongruenz von Betroffenheit und Mitwirkungsrechten}

Der für die politische Philosophie von Kant zentrale Begriff ist der der Republik. Diese ist dadurch gekennzeichnet, dass „[d]ie gesetzgebende Gewalt ... nur dem vereinigten Willen des Volkes zukommen [kann]. "64 Kants Grundidee ist also, dass die Rechtsunterworfenen als Rechtsurheber begriffen werden. ${ }^{65}$ Der Ursprung normativer Legitimation liegt in der gleichen Freiheit der einzelnen Menschen. ${ }^{66}$ Allerdings hat er die aktive Staatsbürgerschaft auf wirtschaftlich unabhängige Bürger begrenzt. ${ }^{67}$ Dies ist jedoch eine historisch kontingente Einschränkung. ${ }^{68}$ Heute besteht dagegen Konsens, dass Demokratie dadurch gekennzeichnet ist, dass die Entscheidungsmacht auf der Grundlage von Regeln einer sehr hohen Zahl von Gruppenmit-

63 S. Kadelbach, Unionsbürgerschaft, in: A. v. Bogdandy/J. Bast (Hrsg.), Europäisches Verfassungsrecht, 2. Aufl., Berlin u.a. 2009, S. 611 (629 f.); s.a. Schönberger, Unionsbürger (Fn. 2), S. 499: „föderativ gegliederte Gesamtheit der Unionsbürger“; anders BVerfGE 123, 267 (376), wonach aus Art. 14 Abs. 2 UAbs. 1 S. 3 EUV folgt, dass die Repräsentation an der Staatsangehörigkeit anknüpft, was aber im Wortlaut nicht nachvollziehbar ist; krit. dazu auch C.D. Classen, Legitime Stärkung des Bundestages oder verfassungsrechtliches Prokrustesbett?, JZ 2009, S. 881 (883); Murkens, Sovereignty (Fn. 6), S. 545.

64 I. Kant, Die Metaphysik der Sitten, in: W. Weischedel (Hrsg.), Werkausgabe, Frankfurt am Main 1968, Bd. VIII, S. 309 (432).

65 Höffe, Globalisierung (Fn. 18), S. 107 f.; Benhabib, Rechte (Fn. 33), S. 30.

66 Keil, Ausländerwahlrechte (Fn. 24), S. 272 ff.

67 Kant, Metaphysik (Fn.64), S. 432 ff.; zur Erläuterung vgl. Keil, Ausländerwahlrechte (Fn. 24), S. 223 ff.; P. Cancik, Selbst ist das Volk? - Der Ruf nach „Volksthümlichkeit der Verwaltung“ in der ersten Hälfte des 19. Jahrhunderts, Der Staat 43 (2004), S. 298 (306 ff.).

68 G. Frankenberg, Die Verfassung der Republik, Baden-Baden 1996, S. 131. 
gliedern zukommt. ${ }^{69}$ Der Ausschluss vom Wahlrecht kann nur noch mit wenigen Gründen, insbesondere Alter und Einsichtsfähigkeit, begründet werden.

Das Diskursprinzip nach Habermas verweist ebenfalls darauf, dass diejenigen Handlungsnormen gültig sind, „denen alle möglicherweise Betroffenen als Teilnehmer an rationalen Diskursen zustimmen könnten “. ${ }^{70}$ Die Bürger eines demokratischen Rechtsstaats verstehen sich danach als Autoren der Gesetze, denen sie als Adressaten gehorchen müssen. ${ }^{71}$ Die in eine politische Öffentlichkeit eingebetteten Partizipationsverfahren gewährleisten die Rückbindung der staatlichen Regelungen an die Selbstbestimmung der von ihnen Betroffenen.

Dieses Prinzip der „Selbsteinwirkung“ der Gesellschaft war historisch lange Zeit nur auf territorial begrenzte Gruppen bezogen, die durch eine gemeinsame Staatsangehörigkeit definiert und damit von anderen Gruppen abgegrenzt sind. ${ }^{72}$ Das demokratisch-deliberative Verständnis der Rechtserzeugung verlangt jedoch, potentiell alle Rechtsbetroffenen zu inkludieren, d.h. als „universale Rechtsgenossen“ anzuerkennen. ${ }^{73}$ Damit werden Defizite deutlich, die zwar nicht grundsätzlich neu sind, aber in der modernen Weltordnung immer größere Bedeutung gewinnen.

\section{Die Diskrepanz zwischen Rechtsautorschaft und Rechtsbetroffenheit}

Zum einen sind in vielen Staaten Ausländer, die der Rechtsordnung ihres Wohnsitzstaates unterworfen sind, ganz oder teilweise von den demokratischen Mitwirkungsrechten ausgeschlossen. Dies führt zu einer internen Diskrepanz zwischen Rechtsautorschaft und Rechtsbetroffenheit, die hier nicht weiterverfolgt werden kann. Zum anderen haben nationale politische Entscheidungen aufgrund der globalen Vernetzung nicht nur der ökonomischen Systeme sondern auch der ökologischen Lebensgrundlagen exterritoriale Auswirkungen, die teilweise gravierend sind. Damit fehlt es ebenfalls an der Abstimmung der Kreise der Betroffenen und der Entscheidungsträger, ${ }^{74}$ was als externe Diskrepanz bezeichnet werden kann.

Die externe Diskrepanz zwischen Betroffenheit und Mitwirkung ist die Folge von vielen internationalen Entwicklungen, die von wenigen Staaten ausgehen und konkrete Auswirkungen auf Menschen in anderen Ländern haben, ohne dass diese in

69 N. Bobbio, Die Zukunft der Demokratie, Berlin 1988, S. 7 (8 f.).

70 J. Habermas, Faktizität und Geltung, Frankfurt am Main 1992, S. 138; vgl. auch die ausführliche Rekonstruktion bei D. Gaus, Der Sinn von Demokratie, Frankfurt am Main 2009, S. $21 \mathrm{ff}$.

71 J. Habermas, Die postnationale Konstellation und die Zukunft der Demokratie, in: ders., Postnationale Konstellation (Fn. 1), S. 91 (152 f.).

72 Habermas, Zukunft (Fn. 71), S. 98 f.

73 N. Fraser, Die Transnationalisierung der Öffentlichkeit. Legitimität und Effektivität der öffentlichen Meinung in einer postwestfälischen Welt, in: Niesen/Herborth (Hrsg.), Anarchie (Fn. 20), S. 224 (249); T. Hitzel-Cassagnes/N. Meisterhans, Konstitutionalisierungsperspektiven eines fragmentierten Weltrechts, in: Brunkhorst (Hrsg.), Weltgesellschaft (Fn. 31), S. 159 (177).

74 Held, Demokratie (Fn. 9), S. $154 \mathrm{ff}$. 
den bestehenden internationalen Institutionen faktisch relevante Einwirkungsmöglichkeiten hätten. Ein einleuchtendes Beispiel ist der Klimawandel, denn sowohl die Verursachung als auch die Folgen der Klimaerwärmung sind international sehr ungleich verteilt. Nach dem letzten Bericht des UN-Sachverständigengremiums International Panel on Climate Change ${ }^{75}$ ist die $\mathrm{CO}^{2}$-Konzentration in der Erdatmosphäre seit 1750 um $35 \%$ angestiegen, die jährliche Emission hat von 1970 bis 2004 um $80 \%$ zugenommen. Die globale Durchschnittstemperatur ist von 1905 bis 2005 um ca. 0,74 Grad Celsius angestiegen. Extreme Wetterereignisse, die zu Überschwemmungen oder verstärkter Trockenheit führen, nehmen zu, der Meeresspiegel steigt kontinuierlich an. Um die Zunahme der Treibhausgase zu begrenzen, ist bis 2050 ein weitgehender Ausstieg aus der Verwendung von fossilen Brennstoffen erforderlich. Während die Industriestaaten für den größten Anteil der Emissionen verantwortlich sind, treffen die negativen Folgen in Form von Trockenheit, Erosion, Extremwetterereignissen u.a. vor allem die Menschen in Afrika und Asien. ${ }^{76}$ Insbesondere nehmen Überschwemmungen zu, durch die z.B. in Südasien und den Anliegerstaaten der Karibik Tausende ihr Leben und Millionen ihr Obdach verlieren.

Die negativen Umweltfolgen der Wirtschaftsweise der hochentwickelten Länder haben eine globale Reichweite. Den Menschen in den armen Ländern stehen jedoch keine Möglichkeiten zur Verfügung, auf die politischen Entscheidungen in den reichen Ländern effektiv Einfluss zu nehmen. Nach dem klassischen Völkerrecht steht es jedem Staat frei, vertragliche Verpflichtungen zu übernehmen. Deshalb konnten sich die USA als größter $\mathrm{CO}^{2}$-Emittent weigern, das Kyoto-Protokoll zu ratifizieren. Appelle an die öffentliche Meinung in den Industriestaaten und Einflussnahmen über die Beteiligung von Nicht-Regierungsorganisationen auf den internationalen Konferenzen haben nur eine sehr begrenzte Wirkung, wie sich zuletzt am Scheitern der Klimakonferenz von Kopenhagen gezeigt hat. Auch wenn die Analysen über die Ursachen dafür, dass keine Einigkeit über ein rechtsverbindliches Abkommen erzielt werden konnte, auseinandergehen, ${ }^{77}$ spricht viel dafür, dass die Interessengegensätze zwischen den verschiedenen Staatengruppen zu stark sind, um im Konsens ein hinreichend ehrgeiziges und durchführbares Abkommen zur Verminderung von Treibhausgasemissionen zu vereinbaren. Insbesondere in den USA und in China fehlt nach wie vor ein breites gesellschaftliches Problembewusstsein über Ursachen und Folgen des Klimawandels. ${ }^{78}$

75 IPCC, Climate Change 2007, Synthesis Report, Summary for Policymakers, S. 2; abrufbar unter http:// www.ipcc.ch (14.10.2010).

76 V. Oschmann/A. Rostankowski, Das Internationale Klimaschutzrecht nach Kopenhagen, ZUR 2010, S. 59 (64) m.w.N.

77 Vgl. die Beiträge von J. Depledge, R. L. Ottinger und J. MacNeill, UNFCCC / CP.15 - The Outcome from Copenhagen in: Environmental Policy and Law 40/1 (2010), S. 17, 22, 24.

78 MacNeill, Copenhagen (Fn. 77), S. 25. 
Das Erfordernis der Einstimmigkeit auf internationaler Ebene verletzt jedoch das Gleichheitsprinzip und führt zu oligarchischen Verhältnissen. ${ }^{79}$ Wenn jeder Beteiligte eine Entscheidung blockieren kann, erhalten Sonderinteressen und Querulanten ein Vetorecht. ${ }^{80}$ Es ist in der politischen Theorie anerkannt, dass die effektive gemeinsame Problembewältigung durch kollektiv verbindliche Entscheidungen nur auf der Grundlage des Mehrheitsprinzips erfolgen kann und es für deren Legitimität ausreicht, wenn alle Beteiligten eine gleiche Mitwirkungschance haben. ${ }^{81}$ Einer der zentralen Gründe für die Rechtfertigung der demokratischen Verfahren des Nationalstaats ist, dass nur so der Legitimationsbedarf von Verteilungsentscheidungen befriedigt werden kann. ${ }^{82}$ Die Umverteilung von Ressourcen, wie sie für den modernen Sozialstaat kennzeichnend ist, wie sie aber ebenfalls für die aus ökologischen Gründen notwendigen Änderungen der Produktionsformen erforderlich ist, kann nicht im Konsens erfolgen. Wenn aber freiwillige Verpflichtungen heute nicht mehr ausreichen, um die erforderlichen internationalen Regelungen der dringenden ökologischen, ökonomischen und sozialen Probleme zu erzielen, müssen majoritäre Verfahren auch im globalen Maßstab installiert werden. ${ }^{83}$

\section{Demokratie als individuelles Recht in Mehrebenensystemen}

Die interne und die externe Diskrepanz zwischen Betroffenheit und Mitwirkungsrecht stellt nicht nur ein politisches Defizit dar. Es hat auch eine menschenrechtliche Dimension, die bisher kaum thematisiert worden ist, da die Menschenrechte zumeist einseitig nur als rechtsstaatliches Instrument angesehen werden. Die meisten Rechte, die in den internationalen Menschenrechtspakten, der Europäischen Menschenrechtskonvention und der Charta der Grundrechte der Europäischen Union garantiert sind, knüpfen nicht an der Staatsangehörigkeit an, sondern sind Jedermannsrechte. Auch die Grundrechte des deutschen Grundgesetzes sind überwiegend nicht auf Deutsche beschränkt. ${ }^{84}$

Der praktische Wert der Menschenrechte ergibt sich aber nicht allein aus den Gewährleistungen der Kataloge auf den verschiedenen Ebenen. Ob sich ein Individuum in einem konkreten Fall erfolgreich auf ein Menschenrecht berufen kann, hängt viel-

79 A. Peters, Dual Democracy, in: J. Klabbers/A. Peters/G. Ulfstein, The Constitutionalization of International Law, Oxford: Oxford University Press 2009, S. 263 (286 ff.).

80 Vgl. Held, Demokratie (Fn. 9), S. 265 f.; E. O. Eriksen, Deliberation und demokratische Legitimität in der EU - Zwischen Konsens und Kompromiss, in: Niesen/Herborth (Hrsg.), Anarchie (Fn. 20), S. 294 (319).

81 Vgl. z.B. Gaus, Sinn (Fn. 70), S. 249 ff., mit Bezug auf Scharpf.

82 Habermas, Weltgesellschaft (Fn. 14), S. 358.

83 Bryde, Constitutionalism (Fn. 13), S. 116 ff.; Peters, Dual Democracy (Fn. 79), S. 263 ff.; ähnlich bereits D. Archibugi, Principles of Cosmopolitan Democracy, in: D. Archibugi/D. Held/M. Köhler (Hrsg.), Re-Imagining Political Community, Stanford: Stanford University Press 1998, S. 198 ff.

84 Vgl. zur Unterscheidung von Menschen- und Bürgerrechten z.B. H. Dreier, in: ders. (Hrsg.), GG, Kommentar, Bd. 1, 2. Aufl. 2004, Vorb. Rn. 71 ff. 
mehr entscheidend davon ab, welchen Schranken es unterliegt. Die meisten Rechtsgarantien enthalten schon in ihrem Wortlaut den Hinweis auf die Möglichkeit gesetzlicher Beschränkungen. Die Schöpfer der EU-Grundrechtecharta haben in Art. 52 den Weg einer allgemeinen Schrankenklausel gewählt. ${ }^{85}$ Das Bundesverfassungsgericht hat bekanntlich auch für die normtextlich vorbehaltslos garantierten Grundrechte die Möglichkeit der Begrenzung durch verfassungsimmanente Schranken anerkannt. ${ }^{86}$ Liegt aber eine verfassungskonforme Beschränkung eines Menschenrechts durch ein Gesetz vor, so bleibt die Berufung auf dieses Recht erfolglos, auch wenn der Schutzbereich eröffnet ist. Welche Schranken tatsächlich bestehen, wie weitgehend das Grundrecht also in seiner Reichweite beschränkt ist, ist eine Entscheidung, die nicht von der Verfassung vorgegeben ist, sondern dem demokratischen Prozess überlassen ist, sofern nicht der Kernbereich betroffen ist. Eher noch größer ist der Entscheidungsspielraum bei den Rechten, die einer Ausgestaltung durch den Gesetzgeber bedürfen, wie etwa im Fall des Eigentums. ${ }^{87}$

Besonders wichtig ist die Rolle des Gesetzes bei der Schutzdimension der Menschenrechte. Soweit sich aus einzelnen Rechten die Pflicht zum Schutz gegen Eingriffe durch private Dritte ergibt, richtet sie sich an den jeweils zuständigen Gesetzgeber. Da sich regelmäßig Kollisionen mit den Grundrechten dieser Dritten ergeben, bedarf es einer Abwägung, die in die genuine Zuständigkeit der Legislative fällt. ${ }^{88}$ Ihre vom Bundesverfassungsgericht oft betonte Einschätzungsprärogative ${ }^{89}$ verweist folglich ebenfalls auf den demokratischen Entscheidungsprozess.

Damit wird deutlich, dass die gerade in der deutschen Diskussion häufig anzutreffende scharfe Trennung zwischen Rechtsstaat und Demokratie unzutreffend ist. Die Menschenrechte stehen nicht in Konkurrenz zur Volkssouveränität, sondern sind die konstitutive Bedingung ihrer Funktionsfähigkeit. ${ }^{90}$ Die allermeisten Konflikte bei der Anwendung von Menschenrechten liegen außerhalb ihres jeweiligen Kernbereichs und damit im Zugriffsbereich des Gesetzgebers. Folglich müssen die Inhaber der Menschenrechte, d.h. alle natürlichen Personen, ein originäres Interesse daran haben, an der Gestaltung der Gesetze mitzuwirken, aus denen sich die Schranken bzw. die Schutzwirkung ihrer Menschenrechte ergibt.

Die externe Diskrepanz kann sich unter bestimmten Voraussetzungen als menschenrechtliches Problem erweisen. Am deutlichsten ist dies bei Militäreinsätzen in frem-

85 Dazu B. Fassbender, Der einheitliche Gesetzesvorbehalt der EU-Grundrechtecharta und seine Bedeutung für die deutsche Rechtsordnung, NVwZ 2010, S. 1049 ff.

86 Grundlegend BVerfGE 28, 243 (260 f.).

87 Dazu J. Wieland, in: Dreier (Hrsg.), GG (Fn. 84), Art. 14 Rn. 25 ff.

88 Vgl. z.B. Dreier (Fn. 84), Vorb. Rn. 101 ff., m.w.N.; G. Webber, The Negotiable Constitution, Cambridge: Cambridge University Press, 2009, S. $147 \mathrm{ff}$.

89 BVerfGE 39, 1 (44); 77, 170 (214); 85, 191 (212).

90 J. Habermas, Volkssouveränität als Verfahren, Merkur 1989, S. 465 (469). 
den Staaten, die nicht der Eroberung dienen sollen, sondern heute meist mit dem Schutz der Menschenrechte begründet werden. ${ }^{91}$ Die Verunklarung der Grenze zwischen militärischen und polizeilichen Zwecken bringt eine Form des Einsatzes von Gewaltmitteln mit sich, die häufig auch zur Verletzung von Rechten der örtlichen Zivilbevölkerung führt. Dies ist der deutschen Öffentlichkeit etwa beim missglückten Einsatz der ISAF-Kräfte in Kundus am 4.9.2009 deutlich geworden, der knapp hundert Zivilisten das Leben gekostet hat. Damit liegt zweifellos ein Eingriff in das Recht auf Leben aus Art. 6 Abs. 1 des Internationalen Pakts über bürgerliche und politische Rechte (IPBürgR) vor, dessen Rechtfertigung strittig ist. ${ }^{92}$ Die Vertreter Afghanistans hatten und haben aber ganz offensichtlich keine ernsthafte Chance, auf die Einsatzentscheidungen der NATO-Streitkräfte Einfluss zu nehmen. Für diese Einsätze fehlen zudem nach wie vor abstrakt-generelle Regeln des Völkerrechts, in denen die Voraussetzungen und die Entscheidungs- und Kontrollverfahren für sog. humanitäre Einsätze hinreichend präzise festgelegt worden wären. ${ }^{93}$ Das gleiche Defizit besteht im Übrigen auf nationaler Ebene in Deutschland, denn für die Auslandseinsätze der Bundeswehr gibt es keinerlei gesetzliche Vorgaben.

Ein zweites, menschenrechtlich schwerer zu fassendes Beispiel sind die globalen Klimaveränderungen. Durch die Auswirkungen des Treibhauseffekts werden Menschenrechte der Betroffenen wie das Recht auf Gesundheit und Eigentum, in vielen Fällen auch das Recht auf Leben beeinträchtigt. ${ }^{94}$ Dadurch sind das in Art. 6 Abs. 1 IPBürgR gewährleistete Recht auf Leben sowie die in Art. 12 Abs. 1 bzw. Art. 11 des Internationalen Pakts über wirtschaftliche, soziale und kulturelle Rechte verankerten Rechte auf Gesundheit und auf gesunde Ernährung berührt. Grundsätzlich ist auch im völkerrechtlichen Menschenrechtsschutz das Bestehen von Schutzpflichten anerkannt. ${ }^{95}$ In welcher Art eine anteilige Verantwortung für Treibhausgasemissionen besteht und wann der erforderliche Sorgfaltsmaßstab verletzt wird, ist allerdings noch weitgehend ungeklärt. ${ }^{96}$

91 Umfassend zur völkerrechtlichen Diskussion C. Verlage, Responsibility to Protect, Tübingen 2009.

92 Zur strafrechtlichen Beurteilung vgl. W. Kaleck/A. Schüller/D. Steiger, Tarnen und Täuschen. Die deutschen Strafverfolgungsbehörden und der Fall des Luftangriffs bei Kundus, KJ 43 (2010), S. 270 ff.

93 Großzügiger D. Thym, Zwischen „Krieg “ und „Frieden“: Rechtsmaßstäbe für operatives Handeln der Bundeswehr im Ausland, DÖV 2010, S. 621 ff., der aber auch darauf hinweist, dass die deutschen Polizeigesetze präziser sind als die Regeln des humanitären Völkerrechts und der internationalen Menschenrechtspakte.

94 F. Ekardt, Wird die Demokratie ungerecht?, München 2007, S. 84.

95 Vgl. z.B. zur Rechtsprechung des EGMR C. Grabenwarter, Europäische Menschenrechtskonvention, 4. Aufl., München 2009, $\mathbb{S} 19$ Rn. 7 ff.; R. Schmidt-Radefeldt, Ökologische Menschenrechte, 2000, S. 55 ff.; zu Schutzpflichten im Bereich der UN-Pakte vgl. W. Kälin/J. Künzli, Universeller Menschenrechtsschutz, 2. Aufl., Basel: Helbing Lichtenhahn 2008, S. 118 ff.; s.a. C. Vedder, Die allgemeinen UN-Menschenrechtspakte und ihre Verfahren, in: D. Merten/H.-J. Papier (Hrsg.), Handbuch der Grundrechte, Bd. VI/2, Heidelberg 2009, $\$ 174$ Rn. 22, 29, 119.

Oschmann/Rostankowski, Klimaschutzrecht (Fn. 76), S. 65. 
Obwohl die wesentlichen Erkenntnisse seit etwa zwanzig Jahren vorliegen, haben die meisten Industriestaaten zu wenig unternommen, um den Treibhauseffekt zu bremsen. Die Zielvorgaben des Kyoto-Protokolls sind unzureichend, ${ }^{97}$ ganz abgesehen davon, dass die USA und China als die beiden größten Emittenten aus unterschiedlichen Gründen dadurch nicht zu Reduktionsmaßnahmen verpflichtet werden. Auch wenn sich aufgrund der abstrakten und globalen Kausalzusammenhänge nicht bestimmen lässt, welche einzelnen Individuen in welchem Zeitrahmen durch Klimaveränderungen konkret in ihren Rechten beeinträchtigt werden, ${ }^{98}$ besteht eine vorrangige Verantwortlichkeit der Industriestaaten für die gestiegene Wahrscheinlichkeit solcher Rechtsbeeinträchtigungen. Wann sich eine solche Risikoerhöhung zu einer Handlungspflicht des Gesetzgebers verdichtet, gegen die Verursacher vorzugehen, ist im nationalen Umweltverfassungsrecht umstritten. ${ }^{99}$ Auf internationaler Ebene fehlt es aber schon an einer Rechtsarena, in der eine entsprechende Auseinandersetzung geführt werden könnte. Die Chance der hauptsächlich betroffenen Staaten, die Interessen ihrer Bevölkerung in internationalen Verhandlungen in ausreichendem Umfang durchzusetzen, sind - wie dargestellt - verschwindend gering. Ein globales parlamentarisches Forum, in dem Vertreter der vorrangig betroffenen Regionen ihre Interessen einbringen und möglicherweise durch den Erlass bindender Regelungen durchsetzen könnten, fehlt. Deshalb ist die Schlussfolgerung konsequent, dass die Umsetzung der Kongruenz zwischen Betroffenheit und Mitbestimmung nach der Schaffung neuer transnationaler Gewalten verlangt. ${ }^{100}$

Dass diese Argumentation über das geltende Recht hinausgeht, liegt auf der Hand. Wenn aus den Menschenrechten zu folgern ist, dass alle Betroffenen an ihrer Ausgestaltung mitwirken können müssen, so dürfte es für die Gewährung des zentralen demokratischen Rechts, des Wahlrechts, nicht auf die Staatsangehörigkeit ankommen. Art. 25 IPBürgR beschränkt die Garantie des Wahlrechts wie des Rechtes zur Teilnahme an der Gestaltung der öffentlichen Angelegenheiten jedoch auf die Staatsbürger. ${ }^{101}$ Interessanterweise fehlt dagegen eine entsprechende ausdrückliche Begrenzung des Kreises der Berechtigten sowohl in Art. 38 GG als auch in Art. 3 1. ZP. EMRK. Obwohl nach Art. 1 EMRK, auf den Art. 5 1. ZP. EMRK verweist, die Rechte allen der Hoheitsgewalt der Vertragsparteien unterstehenden Personen zustehen, wird das Wahlrecht unter Verweis auf die Verfassungstradition auf Staats-

97 Ekardt, Demokratie (Fn. 94), S. 19 f.

98 T. Groß, Welche Klimaschutzpflichten ergeben sich aus Art. 20 a GG?, ZUR 2009, S. 364 (366).

99 Zusammenfassend etwa R. Schmidt/W. Kahl, Umweltrecht, 8. Aufl., München 2010, 22 Rn. 12 ff.

100 J. Kokott, Die Staatsrechtslehre und die Veränderung ihres Gegenstands, VVDStRL 63 (2004), S. 7 (31); Fraser, Öffentlichkeit (Fn. 73), S. 224 (252).

101 Vgl. dazu M. Nowak, CCPR Commentary, 2. Aufl., Kehl u.a. 2005, Art. 25 Rn. 8. 
angehörige beschränkt. ${ }^{102}$ Ebenfalls fehlt in Art. 38 GG eine Eingrenzung des Kreises der Grundrechtsträger. Hier wird aus der Formulierung in Art. 38 Abs. 1 S. 2 GG, wonach die Abgeordneten „Vertreter des ganzen Volkes“ sind, abgeleitet, dass es sich um ein Deutschenrecht handelt. ${ }^{103}$

Wenn man den Volksbegriff von seiner nationalstaatlichen Verengung löst, so verliert die traditionelle Beschränkung politischer Mitwirkungsrechte auf die Staatsbürger ihre Selbstverständlichkeit. Vielmehr wird deutlich, dass zwischen universellen Menschenrechten, die für jeden unabhängig von der Staatsangehörigkeit gelten, und der jeweils partikularen nationalen Identität ein Spannungsverhältnis besteht. ${ }^{104}$ „Der Bedarf nach demokratischer Inklusion auch jenseits der Staatsgrenzen ist die Konsequenz offener Staatlichkeit. “105

Aus dem Grundprinzip der Demokratie, dass die politischen Gestaltungsrechte an die persönliche Betroffenheit gekoppelt sein müssen, folgt, dass das Recht auf demokratische Mitwirkung ein individuelles Recht ist, das in der Menschenwürde wurzelt. ${ }^{106}$ Der konzeptionelle Bezugspunkt demokratischer Rechte ist die individuelle Selbstbestimmung. ${ }^{107}$ „Demokratie ist positives Recht eines jeden menschlichen Wesens. "108 Wenn aber aufgrund faktischer Entwicklungen die durch ihre Betroffenheit definierten Gruppen und die überkommenen Kollektive, die insbesondere durch die Staatsangehörigkeit definiert sind, immer weiter divergieren, so ergibt sich ein Handlungsbedarf, um die Inkongruenzen zu verringern. Demokratie ist aus dieser Sicht nicht auf Staaten begrenzt, sondern kann auch in supra- und internationalen Organisationen installiert werden. ${ }^{109}$ In einem gestuften Mehrebenensystem können

102 Grabenwarter, Menschenrechtskonvention (Fn. 95), \$23 Rn. 93; L. Wildhaber, in: W. Karl (Hrsg.), Int. Kommentar zur Europäischen Menschenrechtskonvention, Köln, Loseblatt (Stand 2009), Art. 3 1. ZP Rn. 25; anders D. Richter, Das Recht auf freie Wahlen, in: R. Grote/T. Marauhn (Hrsg.), EMRK/GG, Konkordanzkommentar, Tübingen 2006, Kap. 25 Rn. 15, unter Hinweis auf die Freiheit der Konventionsstaaten, ihr Wahlsystem zu bestimmen.

103 So z.B. M. Morlok, in: Dreier (Hrsg.), GG, Bd. 2, 2. Aufl. 2006, Art. 38 Rn. 71; W. Schreiber, in: Friauf/Höfling (Hrsg.), GG (Fn. 40), Art. 38 Rn. 14; relativierend H.-P. Schneider, in: E. Denninger/ W. Hoffmann-Riem/H.-P. Schneider/E. Stein (Hrsg.), AK-GG, 3. Aufl., Neuwied u.a. 2002, Art. 38 Rn. 77.

104 Benhabib, Rechte (Fn. 33), S. 52.

105 Kotzur, Wettbewerbsordnung (Fn. 33), S. 198.

106 P. Häberle, Die Menschenwürde als Grundlage der staatlichen Gemeinschaft, in: J. Isensee/P. Kirchhof (Hrsg.), Handbuch des Staatsrechts, Bd. 1, 1. Aufl. 1987, $\$ 20$ Rn. 66; B.-O. Bryde, Demokratisches Europa und Europäische Demokratie, in: C. Gaitanides/S. Kadelbach/G.C. Rodriguez Iglesias (Hrsg.), Europa und seine Verfassung, FS Zuleeg, Baden-Baden 2005, S. 131 (132); A. v. Bogdan$d y$, Gubernative Rechtsetzung, Tübingen 2000, S. 31 f.; Trute, Legitimation (Fn. 21), Rn. 19; ähnlich H. Dreier, Der freiheitliche Verfassungsstaat als riskante Ordnung, Rechtswissenschaft 2010, S. 11 (16): „Legitimationsgrund der politischen Ordnung ist der einzelne und freie Mensch..."

107 T. Blanke, Antidemokratische Effekte der verfassungsgerichtlichen Demokratietheorie, KJ 31 (1998), S. 452 (457); Groß, Kollegialprinzip (Fn. 21), S. 166.

108 Müller, Weltrecht (Fn. 17), S. 72.

109 So auch C. Möllers, Demokratie - Zumutungen und Versprechen, 2. Aufl., Berlin 2009, S. 82. 
institutionelle Angebote egalitärer Beteiligung auf verschiedenen föderalen Ebenen gemacht werden. ${ }^{110}$

\section{Mögliche Formen der Institutionalisierung}

Wenn man die zunehmende Bedeutung der Internationalen Organisationen als Ausdruck einer entstehenden Weltstaatlichkeit betrachtet, die wie jede Form der Herrschaftsausübung demokratischen Standards entsprechen muss, stellt sich die Frage, wie eine möglichst enge Rückkoppelung an die Betroffenen ermöglicht werden kann. Hierfür wird eine Vielfalt von Formen der Institutionalisierung diskutiert. ${ }^{111} \mathrm{Im}$ Folgenden werden vier Vorschläge analysiert, mit denen demokratische Strukturen oberhalb der nationalen Ebene geschaffen werden sollen. Sie stehen nicht in einem Ausschließungsverhältnis, sondern könnten auch neben- oder nacheinander verwirklicht werden.

\section{Die Verbesserung der Mitwirkung nationaler Parlamente}

Viele Stimmen wollen die demokratische Legitimation des Völkerrechts durch eine Verbesserung der Rückkoppelung an die nationalen Parlamente stärken. ${ }^{112}$ Dabei wird insbesondere auf die Regelungen in Bezug auf die EU in Art. 23 GG und den Ausführungsgesetzen verwiesen. ${ }^{113}$ Eine frühzeitige und umfassende Einbeziehung der Parlamentarier kann die Machtballung bei der nationalen Exekutive verringern.

Die entscheidenden Strukturprobleme der internationalen Institutionen können dadurch aber nicht gelöst werden. Insbesondere wird das Blockadepotential des Einstimmigkeitsprinzips nicht verringert, sondern eher noch verstärkt. Die Notwendigkeit, dass die Verhandlungsführer ständig auf die Meinungsbildung in ihren nationalen Parlamenten Rücksicht nehmen, kann die Dynamik internationaler Verhandlungen erheblich beeinträchtigen. Deutlich wird dieser Konflikt etwa im Fall der Vereinigten Staaten, wo das Erfordernis einer Zwei-Drittel-Mehrheit im Senat für die Ratifikation internationaler Verträge oft zu einer Handlungsunfähigkeit des Präsidenten in internationalen Verhandlungen führt. Die noch weitergehende Option eines imperativen Mandats des Parlaments gegenüber der Regierung, wie es etwa für

110 C. Möllers, Expressive versus repräsentative Demokratie, in: R. Kreide/A. Niederberger (Hrsg.), Transnationale Verrechtlichung, Frankfurt am Main 2008, S. 160 (178).

111 Vgl. z.B. den Überblick bei McGrew, Grenzen (Fn. 8), S. 388 ff.; s.a. M. Albert/R. Schmalz-Bruns, Antinomien der Global Governance, in: Brunkhorst (Hrsg.), Weltgesellschaft (Fn. 31), S. 57 ff.

112 Vgl. z.B. Seiler, Verfassungsstaat (Fn. 29), S. 356 f.; W. Kahl, Parlamentarische Steuerung der internationalen Verwaltungsvorgänge, in: H.-H.Trute/T. Groß/C. Röhl/C. Möllers (Hrsg.), Allgemeines Verwaltungsrecht - zur Tragfähigkeit eines Konzepts, Tübingen 2008, S. 71 (86 ff.); Poscher, Globalisierung (Fn. 2), S. 160 (186 ff.); zurückhaltend M. Ruffert, Perspektiven des Internationalen Verwaltungsrechts, in: C. Möllers/A. Voßkuhle/C. Walter (Hrsg.), Internationales Verwaltungsrecht, Tübingen 2007, S. 395 (408).

113 Classen, Legitimation (Fn. 2), S. 117. 
die Stimmabgabe im Rat der EU diskutiert wird, ${ }^{114}$ kann sich auch Fessel erweisen und die Dauer der Beratungen in die Länge ziehen.

\section{Die Verbesserung der Transparenz}

Ein zweiter, bereits oft vorgebrachter Vorschlag, der teilweise auch schon verwirklicht wird, ist die Verbesserung der Transparenz internationaler Verhandlungen. ${ }^{115}$ Eine Rückbindung der Entscheidungsprozesse an eine weitere Öffentlichkeit kann durch die frühzeitige und begleitende Information der Medien sowie die Nutzung des Internets als Kommunikationsmedium erfolgen. Außerdem können Vertreter von Nicht-Regierungsorganisationen an internationalen Konferenzen teilnehmen und spezifische Interessen und Informationen in die Verhandlungen einbringen. ${ }^{116} \mathrm{Ge}$ rade im internationalen Umweltrecht spielen private Umweltorganisationen bereits eine wichtige Rolle. ${ }^{117}$ Im Beispielsfall der Klimakonferenz von Kopenhagen wurden ihre Vertreter allerdings in der entscheidenden Phase von den Verhandlungen ausgeschlossen. Die internationalen Menschenrechtsinstitutionen sind wesentlich auf Informationen durch private Menschenrechtsorganisationen angewiesen, um ihre Kontrollfunktion erfüllen zu können. ${ }^{118}$

Eine solche Verbesserung der informellen Partizipation und der Transparenz kann man grundsätzlich als Mittel der Demokratisierung einordnen. Es darf aber nicht übersehen werden, dass die Vertreter privater Organisationen nicht ausreichend legitimiert sind, um eine eigenständige Legitimation verbindlicher Entscheidungen zu liefern, denn sie sind keine Repräsentanten der Bürger insgesamt, sondern nur von Interessengruppen. ${ }^{119}$ Die Ermöglichung von Verfahren der Deliberation auf inter-

114 Ausführlich C. Rath, Entscheidungspotentiale des Deutschen Bundestags in EU-Angelegenheiten, Baden-Baden 2001, S. $103 \mathrm{ff}$.

115 Habermas, Zukunft (Fn. 71), S. 166 f.; S. Marks, The Riddle of All Constitutions, Oxford u.a.: Oxford University Press 2000, S. 113 f.; Müller, Weltrecht (Fn. 17), S. 79 ff.; Classen, Legitimation (Fn. 2), S. 116; J. Bast, Das Demokratiedefizit fragmentierter Internationalisierung, in: Brunkhorst (Hrsg.), Weltgesellschaft (Fn. 31), S. 185 (190 f.); J. v. Bernstorff, Procedures of Decision-Making and the Role of Law in International Organizations, in: v. Bogdandy et al. (Hrsg.), Exercise (Fn. 13), S. 777 (799f.).

116 Vgl. z.B. A. Peters, Membership in the Global Constitutional Community, in: Klabbers et al. (Fn. 79), S. 153 (219 ff.).

117 B.-O. Bryde, Grenzüberschreitende Umweltverantwortung und ökologische Leistungsfähigkeit der Demokratie, in: K. Lange (Hrsg.), Gesamtverantwortung statt Verantwortungsparzellierung im Umweltrecht, Baden-Baden 1997, S. 75 (84).

118 D. Beetham, Human Rights and Cosmopolitan Democracy, in: Archibugi et al. (Hrsg.), Re-Imagining (Fn. 83), S. 58 (63 f.); Bryde, Constitutionalism (Fn. 13), S. 118 f.

119 P. Nanz/J. Steffek, Zivilgesellschaftliche Partizipation und die Demokratisierung internationalen Regierens, in: Niesen/Herborth (Hrsg.), Anarchie (Fn. 20), S. 87 (105); Sommermann, Demokratie (Fn. 15), S. 1063; Poscher, Globalisierung (Fn. 2), S. 175 f.; Möllers, Demokratie (Fn. 109), S. 99 f. 
nationaler Ebene kann kein Ersatz für die Institutionalisierung egalitärer Mitbestimmungsrechte sein. ${ }^{120}$

\section{Die Schaffung einer globalen parlamentarischen Versammlung}

In der Tradition nationalstaatlicher Demokratie kommt gewählten Volksvertretungen eine zentrale Rolle zu. Mit Ausnahme des Europäischen Parlaments gibt es dagegen in internationalen Organisationen bisher nur indirekt gewählte parlamentarische Versammlungen, deren Mitglieder von den Parlamenten der Mitgliedstaaten bestimmt werden. Inzwischen bestehen über 40 verschiedene, überwiegend regionale Parlamentarische Versammlungen. ${ }^{121}$ Die einzige globale Institution, die Interparlamentarische Union (IPU), ist eine Vereinigung nationaler Parlamente mit inzwischen 155 Mitgliedern. ${ }^{122}$ Sie ist an keine bestehende internationale Organisation angebunden, versteht sich aber als Partner und Unterstützer der UN. Sie könnte zu einem Parlament der Vereinten Nationen weiterentwickelt werden. ${ }^{123}$ Daneben gibt es auch konkrete Vorschläge für eine Parlamentarisierung der WTO ${ }^{124}$ sowie von Weltbank und IWF. 125

Ein sehr konkreter Vorschlag zur Schaffung einer zweiten Kammer der Vereinten Nationen wurde bereits in den 1980er Jahren erarbeitet. ${ }^{126}$ Seit 2007 wirbt eine internationale Kampagne für eine Parlamentarische Versammlung bei den Vereinten Nationen. ${ }^{127}$ Sie soll nach ihrer Errichtung von einem lediglich beratenden Gremium zu einem Weltparlament mit Informations-, Partizipations- und Kontrollrechten ausgebaut werden. Allerdings findet man im Aufruf keine konkreten Angaben über die Sitzverteilung und darüber, wie mit Mitgliedstaaten umgegangen werden soll, die selbst keine Demokratien sind.

Der entscheidende Mangel einer solchen Versammlung liegt darin, dass ihr keine verbindlichen Entscheidungsbefugnisse zustehen, so dass sie keine wirksame Antwort auf das Problemlösungsdefizit auf internationaler Ebene sein kann. Parlamen-

120 J. Habermas, Kommunikative Rationalität und grenzüberschreitende Politik: eine Replik, in: Niesen/ Herborth (Hrsg.), Anarchie (Fn. 20), S. 406 (438); P. Niesen, Deliberation oder Demokratie?, in: Kreide/Niederberger (Hrsg.), Verrechtlichung (Fn. 110), S. 240 (254); J. v. Bernstorff, Zivilgesellschaftliche Partizipation in Internationalen Organisationen: Form globaler Demokratie oder Baustein westlicher Expertenherrschaft, in: Brunkhorst (Hrsg.), Weltgesellschaft (Fn. 31), S. 276 ff.; Peters, Dual Democracy (Fn. 79), S. 270 f.

121 Vgl. den Überblick bei S. Marschall, Transnationale Repräsentation in Parlamentarischen Versammlungen, Baden-Baden 2005, S. $137 \mathrm{ff}$.

122 http://www.ipu.org/english/membshp.htm.

123 Vgl. C. Zöpel, Die Vereinten Nationen und die Parlamente (II), Vereinte Nationen 2005, S. 145 ff.

124 Vgl. das Modell von M. Krajewski, Zur Demokratisierung von Global Economic Governance, in: Brunkhorst (Hrsg.), Weltgesellschaft (Fn. 31), S. 215 (226 ff.).

125 Vgl. Knieper, Souveränität (Fn. 33), S. 192.

126 J.J. Segall, A UN Second Assembly, in: F. Barnaby (Hrsg.), Building a More Democratic UNITED NATIONS, London: Cass 1991, S. $93 \mathrm{ff}$.

127 http://de.unpacampaign.org/index.php (14.10.2010). 
tarische Versammlungen, die nur mittelbar legitimiert sind und denen keine Entscheidungsbefugnisse zukommen, können deshalb nur einen kleinen Beitrag zur Demokratisierung internationaler Organisationen leisten, indem sie die Transparenz verbessern und Kontrolle ermöglichen, indem sie (auch) gesellschaftliche Minderheiteninteressen artikulieren und indem sie gesellschaftliche Akteure mit den Entscheidungsprozessen der internationalen Organisation verknüpfen. ${ }^{128}$

\section{Die Schaffung eines Weltparlaments}

Alle bisher untersuchten Strategien lösen nicht das zentrale Problem der wirksamen egalitären Mitwirkung auf der globalen Ebene. Die einzige Institution zur effektiven Regelung internationaler Verteilungskonflikte wäre ein echtes Weltparlament, das als Organ der Vereinten Nationen mit Gesetzgebungsbefugnissen ausgestattet werden müsste. Diese Idee hat historische Wurzeln, die in das 18. Jahrhundert zurückreichen. ${ }^{129}$ Dabei sind gravierende Probleme der Kompetenzen, der Verteilung der Stimmrechte und der wirksamen Anbindung an eine öffentliche Meinung zu lösen, die hier nur angedeutet werden können. Außerdem stellt natürlich die Integration von Staaten, die intern nicht demokratisch strukturiert sind, ein gravierendes Problem dar.

Habermas hat vorgeschlagen, das Weltparlament auf die Funktionen der Sicherung des Friedens und der Menschenrechte zu beschränken. ${ }^{130}$ Dabei übersieht er aber, dass die Verwirklichung der Menschenrechte eine Vielzahl von Politikfeldern unmittelbar berührt, insbesondere wenn man die sozialen und wirtschaftlichen Rechte einbezieht. So gefährdet, wie erörtert, der Klimawandel die Rechte auf Leben, Gesundheit und Eigentum von Millionen Menschen, so dass eine Regelungsbefugnis für den globalen Umweltschutz dringend notwendig ist. Ebenso bedürfen grundlegende Fragen der Rohstoffnutzung einer internationalen Regelung. Ginge es dagegen nur um eine Überwachung der Einhaltung der Menschenrechte, wäre in erster Linie ein internationaler Gerichtshof notwendig, der analog zum Europäischen Gerichtshof für Menschenrechte Einzelfälle verbindlich entscheiden könnte, aber keine parlamentarische Versammlung.

Besonders problematisch ist die Verteilung der Stimmrechte in einem globalen Parlament. Würde man versuchen, das Postulat des Bundesverfassungsgerichts zu verwirklichen, wonach Entscheidungsbefugnisse eines übernationalen Parlaments zwin-

128 Marschall, Versammlungen (Fn. 121), S. 334 ff.; Sommermann, Demokratie (Fn. 15), S. 1060 f.; Peters, Dual Democracy (Fn. 79), S. 322 ff.

129 Dazu C. Kissling, Repräsentativ-parlamentarische Entwürfe globaler Demokratiegestaltung im Laufe der Zeit, abrufbar unter: http://www.forhistiur.de/zitat/0502kissling.htm (14.10.2010).

130 Habermas, Rationalität (Fn. 120), S. 450 ff. 
gend ein strikt egalitäres Wahlrecht voraussetzen, ${ }^{131}$ so würde die Versammlung aufgrund der immensen Größenunterschiede der jetzigen Mitgliedstaaten unpraktikabel groß. ${ }^{132}$ Auch ein Kompromiss zwischen Staatenegalität und Bevölkerungsproporz würde, wenn die Handlungsfähigkeit des Parlaments erhalten bleiben soll, zu erheblich größeren Verzerrungen führen als im Fall des Europäischen Parlaments. ${ }^{133}$ Eine transnationale demokratische Ordnung kann auf eine ponderierte Vertretung der Mitgliedstaaten nicht verzichten. ${ }^{134}$

Der wohl realistischste Vorschlag ist deshalb eine abgestufte Demokratisierung der globalen Ordnung, in der z.B. auf Kontinente bezogene regionale Organisationen als Zwischeneinheiten zwischen der einzelstaatlichen und der globalen Ebene fungieren. ${ }^{135}$ In der EU existiert bereits ein direkt gewähltes Parlament. Die Parlamente der Mitgliedstaaten der OAS haben sich zu einer parlamentarischen Versammlung zusammengeschlossen, dem Inter-Parliamentary Forum of the Americas (FIPA), ${ }^{136}$ das allerdings kein Organ der OAS ist und keine Entscheidungsbefugnisse hat. Auch Art. 17 des Gründungsvertrages der Afrikanischen Union sieht ein pan-afrikanisches Parlament vor. Seine nähere Ausgestaltung muss durch ein noch nicht verabschiedetes Protokoll erfolgen. In Asien und Ozeanien gibt es bisher noch keine kontinentalen parlamentarischen Strukturen, sie müssten noch geschaffen werden. Immerhin besteht bereits im Rahmen der ASEAN eine interparlamentarische Versammlung. ${ }^{137}$

Nicht zu übersehen ist allerdings, dass die Schaffung eines Weltparlaments allein noch nicht ausreicht, um eine globale demokratische Ordnung zu schaffen. Zum einen muss eine begleitende politische Meinungs- und Willensbildung der Bürger über die Weltinnenpolitik entstehen. ${ }^{138}$ Die Infrastruktur hierfür steht mit den globalen Medienunternehmen, insbesondere aber mit dem dezentralen Internet bereits weitgehend zur Verfügung. Bei einzelnen Themen wie dem Protest gegen die Besetzung des Irak durch die USA oder dem Weltklimagipfel in Kopenhagen sind bereits punktuelle globale Öffentlichkeiten entstanden. Darin ist ein langfristiger Trend zur Internationalisierung der politischen Orientierungen erkennbar, der erweitert und verstetigt werden müsste. ${ }^{139}$

131 BVerfGE 123, 267 (373 ff.); zutreffende Kritik bei C. Schönberger, Lisbon in Karlsruhe - Maastricht's Epigones at Sea, GLJ 2009, S. 1201 (1214 f.); C. Tomuschat, Lisbon - Terminal of the European Integration Process?, ZaöRV 2010, S. 251 (275 f.).

132 Dazu H. Müller, Parlamentarisierung der Weltpolitik - Ein skeptischer Warnruf, in: Kreide/Niederberger (Hrsg.), Verrechtlichung (Fn. 110), S. 137 (141 ff.).

133 Vgl. z.B. die Sitzverteilung nach der Penrose-Methode bei Segall, Assembly (Fn. 126), S. 108 f.

134 Bryde, Europa (Fn. 106), S. 133 f.

135 Höffe, Globalisierung (Fn. 18), S. 306 ff.; ähnlich Habermas, Weltgesellschaft (Fn. 14), S. 337 ff.

136 http://www.e-fipa.org/About/about_en.htm (14.10.2010).

137 http://www.aipasecretariat.org/about/ (14.10.2010).

138 Habermas, Rationalität (Fn. 120), S. 406 (455); Ekardt, Demokratie (Fn. 94), S. 166; U. Mückenberger, Demokratische Einhegung der Globalisierung, KJ 43 (2010), S. 38 ff.

139 Vgl. Held, Demokratie (Fn. 9), S. 147 f. 
Zum anderen blieben die Gesetzesbeschlüsse des Weltparlaments wirkungslos, wenn keine Strukturen zu ihrer exekutiven und judiziellen Implementation geschaffen würden. ${ }^{140}$ Eine vollständig dezentrale Implementation durch kontinentale und nationale Organisationen wäre zwar theoretisch denkbar, würde aber erhebliche Risiken für die Effektivität der globalen Regelungen bergen. Das Beispiel der EU zeigt, dass eine Überwachung der Umsetzung des Europarechts in den Mitgliedstaaten erforderlich ist, die durch die Kommission und den Gerichtshof erfolgt.

\section{E. Einwände}

Das hier zugrundegelegte Demokratieverständnis baut auf der Philosophie von Immanuel Kant auf. Dieser hielt selbst eine globale demokratische Ordnung jedoch nicht für möglich. Das Weltbürgerrecht verstand Kant nur als ein Recht der Fremden auf Gastfreundschaft, aber nicht als Grundlage politischer Gestaltungsrechte. ${ }^{141}$ Seine Begründung dafür, dass die Volkssouveränität auf den staatlichen Rahmen beschränkt bleiben muss und ein Weltstaat ausgeschlossen ist, ist jedoch den damaligen Realbedingungen geschuldet und lässt sich nicht aus seinen normativen Prämissen ableiten. ${ }^{142}$ Damals wie heute werden viele Einwände gegen die Möglichkeit globaler Demokratie vorgebracht, die im Folgenden kurz thematisiert werden. Ob globale demokratische Strukturen in absehbarer Zeit auch durchsetzbar sind, ist eine ganz andere Frage.

\section{Der Einwand der territorialen Größe}

Kant hielt Demokratie in großen Territorien nicht für möglich. ${ }^{143}$ Die Notwendigkeit der Begrenzung eines Gemeinwesens auf einen beschränkten Raum tritt so als Argument neben die personelle Dimension von Demokratie als Organisation abgegrenzter Kollektive.

Allerdings gibt es bereits heute Staaten von sehr großer Ausdehnung, deren demokratische Strukturen zweifellos funktionieren, wie etwa in Kanada, den USA oder Australien. Kants Einwand der Unregierbarkeit ist deshalb als pragmatisches Urteil vor dem Hintergrund der damaligen Verhältnisse einzuordnen, denn gleichzeitig nennt er das Ziel der kontinuierlichen Annäherung an den „Völkerstaat“ als Garant

140 Vgl. Müller, Weltrecht (Fn. 17), S. $91 \mathrm{ff.}$

141 I. Kant, Zum ewigen Frieden, in: Weischedel (Hrsg.), Werkausgabe, Frankfurt am Main 1968, Bd. XI, S. 191 (213 ff.); dazu ausführlich Benhabib, Rechte (Fn. 33), S. 36 ff.

142 Habermas, Rationalität (Fn. 120), S. 440; Keil, Ausländerwahlrechte (Fn. 24), S. 241 ff.; P. Kleingeld, Approaching Perpetual Peace: Kant's Defence of a League of States and his Ideal of a World Federation, European Journal of Philosophy 12 (2004), S. 304 ff.; a.A. Maus, Verfassung (Fn. 20), S. 357 ff.; zum ganzen auch Nils Lange-Bertalot, Weltbürgerliches Völkerrecht, Berlin 2007.

143 Kant, Metaphysik (Fn.64), S. 474; ders., Frieden (Fn. 141), S. 225; ebenso Benhabib, Rechte (Fn. 33), S. $212 \mathrm{f}$. 
des ewigen Friedens. ${ }^{144}$ Heute haben sich aber die modernen Kommunikations- und Transportsysteme so weit entwickelt, dass sie eine Vielzahl globaler Institutionen wie auch eine globale Öffentlichkeit ermöglichen. ${ }^{145} \mathrm{Ob}$ sich gewählte Vertreter oder Diplomaten treffen, ist unter praktischen Gesichtspunkten kein wichtiger Unterschied. Allerdings müssten sich dann auch die zivilgesellschaftlichen Kräfte noch besser vernetzen.

\section{Der Einwand der fehlenden Homogenität}

Ein zentraler Einwand aus der nationalstaatlichen Tradition ist die Behauptung, dass die für die Schaffung eines Gemeinwesens notwendige Solidarität ohne eine hinreichende Homogenität seiner Mitglieder nicht erreicht werden kann. ${ }^{146}$ Auch in der juristischen Diskussion wird die These aufgestellt, dass nur Nationalstaaten die Menschen zu einer Solidargemeinschaft verbinden können. ${ }^{147}$ Die vermittelnden Instanzen zwischen Zivilgesellschaft und internationalen Institutionen fehlen. ${ }^{148}$ Kritiker halten deshalb die Schaffung eines Weltparlaments für „bereits im Ansatz untauglich“, da die integrierende Aufgabe kaum zu leisten sei. ${ }^{149}$

Dagegen spricht jedoch, dass keine Gesellschaft völlig homogen ist. Außerdem ist es unmöglich, einen hinreichenden Homogenitätsgrad abstrakt zu bestimmen. Vielmehr schützen die Grundrechte religiöse, kulturelle oder sprachliche Inhomogenität, wie sich insbesondere aus Art. 3 Abs. 3 GG ergibt. ${ }^{150}$ Dass unterschiedliche Sprachen kein Hinderungsgrund für eine funktionierende Demokratie sind, belegen z.B. in kleinerem Rahmen die Schweiz mit vier Amtssprachen und in größerem Rahmen Indien mit 22 Amtssprachen. Insbesondere ist auch eine demokratische Öffentlichkeit nicht von einer gemeinsamen Sprache abhängig, sondern kann durch ein vernetztes System vielfältiger Teilöffentlichkeiten auch in supranationalen Kontexten wirken. ${ }^{151}$ Die weltweiten Migrationsbewegungen führen in den meisten Staaten zu

144 Kant, Metaphysik (Fn. 64), S. 474; Held, Global Order (Fn. 14), S. 145; Höffe, Globalisierung (Fn. 18), S. $306 \mathrm{f}$.

145 Held, Global Order (Fn. 14), S. $121 \mathrm{ff}$.

146 So etwa Kaufmann, Demokratieprinzip (Fn. 4), S. 50: „demokratische Homogenität im Sinne substantieller Identität"; besonders pointiert die Apotheose der Nation als politischer Gemeinschaft bei U. Haltern, Internationales Verfassungsrecht?, AöR 128 (2003), S. $511 \mathrm{ff}$.

147 Volkmann, Demokratie (Fn. 2), S. 592: „geistige Gemeinschaft“; Müller-Franken, Ausländerwahlrechte (Fn. 24), S. 565, unter Bezug auf „Gemütswerte“; Oliver Eberl/Florian Rödl, Kritische Politische Ökonomie und radikale Demokratietheorie, KJ 2010, S. 416 (419): „Überschaubarkeit der Systeme".

148 T. Christiano, Democratic Legitimacy and International Institutions, in: S. Besson/J. Tasioulas (Hrsg.), The Philosophy of International Law, Oxford u.a.: Oxford University Press 2010, S. 119 (134f.).

149 Classen, Legitimation (Fn. 2), S. 115 f.

150 Bryde, Volksdemokratie (Fn. 18), S. 322; Murkens, Sovereignty (Fn. 6), S. 547.

151 Hanschmann, Homogenität (Fn. 26), S. 236. 
einer größeren religiösen Vielfalt, die zwar konfliktträchtig ist, aber nicht ernsthaft die Akzeptanz der demokratischen Verfahren beeinträchtigt.

Auf der anderen Seite gibt es eine immer engere Verflochtenheit zwischen den Staaten, die in immer mehr Bereichen zur Herausbildung einer internationalen Solidarität führt. Besonders weit entwickelt sind Umverteilungsmechanismen in der Europäischen Union. Auch wenn die zentralen sozialstaatlichen Institutionen in nationaler Verantwortung bleiben, gibt es vielfältige finanzielle Ausgleichs- und Förderverfahren mit einem beträchtlichen Volumen. Besonders deutlich hat sich das im Rahmen der Eurokrise im Frühjahr 2010 zeigt, als die Mitgliedstaaten zur Stabilisierung der gemeinsamen Währung bemerkenswert große Verpflichtungen übernommen haben. ${ }^{152}$ Auf der globalen Ebene gibt es inzwischen eingespielte Mechanismen der Katastrophenhilfe, auch wenn deren Finanzausstattung immer prekär ist. Die politischen Abläufe bei der Einrichtung des Internationalen Strafgerichtshofs werden bereits als Beispiel für die Möglichkeit eines „global demos“ angesehen, da es sich um eine Angelegenheit mit einem hinreichenden „global concern“ handelte, bei dem zunächst enorme Widerstände einzelner Staaten überwunden werden konnten. ${ }^{153}$

Im Übrigen darf die Wechselwirkung zwischen demokratischer Mitwirkung und Inklusion nicht unterschätzt werden. Ein demokratischer Verfassungsstaat kann Lücken in der sozialen Integration durch die politische Partizipation der Bürger schließen und ist deshalb nicht allein auf eine mentale Verwurzelung angewiesen. ${ }^{154}$ Ein kulturell heterogenes supranationales Verfassungsvolk kann durch politisches Verfahren entstehen. ${ }^{155}$ Die Unionsbürgerschaft wird als ein Beispiel für die Anerkennung kultureller Vielfalt auf einem „inter-group level“ angesehen. ${ }^{156}$ Demokratie ist immer offen für eine Vielfalt von politischen und kulturellen Präferenzen. ${ }^{157}$

Zudem ist daran zu erinnern, dass ein Weltparlament nicht dazu gedacht ist, die anderen staatlichen Strukturen zu ersetzen. Die globale Ebene würde ähnlich wie die EU nur als zusätzliche politische Einheit entstehen, nicht als Ersatz für andere Zu-

152 Vgl. A. Fisahn, Griechenland und die Perspektiven der EU, KJ 43 (2010), S. 248 ff.; zum Euro-Stabilisierungsmechanismus-Gesetz vom 22.5.2010 krit. K. Faßbender, Der europäische „Stabilisierungsmechanismus" im Lichte von Unionsrecht und deutschem Verfassungsrecht, NVwZ 2010, S. 799 ff.; s.a. die Ablehnung einer einstweiligen Anordnung durch das BVerfG, NJW 2010, 2418.

153 C. List/M. Koenig-Archibugi, Can There Be a Global Demos? An Agency-Based Approach, Philosophy and Public Affairs 1/38 (2010), S. 76 (103ff.); s.a. N. Deitelhoff, Was vom Tage übrig blieb. Inseln der Überzeugung im vermachteten Alltagsgeschäft internationalen Regierens, in: Niesen/Herborth (Hrsg.), Anarchie (Fn. 20), S. 26 ff.

154 Habermas, Zukunft (Fn. 71), S. 117.

155 M. Neves, Transversale Verfassungen?, in: Brunkhorst (Hrsg.), Weltgesellschaft (Fn. 31 ), S. 75 (88).

156 J. Weiler, The Constitution of Europe, Cambridge: Cambridge University Press 1999, S. $341 \mathrm{ff}$; Gaus, Sinn (Fn. 70), S. 275 ff., weist zutreffend darauf hin, dass sich die Argumentation nicht gegen den Staat, sondern gegen die Nation als Grundlage von Staaten richtet.

157 Held, Global Order (Fn. 14), S. 282; Peters, Dual Democracy (Fn. 79), S. 308 ff. 
gehörigkeiten. ${ }^{158}$ Außerdem wäre das Ziel der Errichtung effektiver internationaler Regelungsmechanismen nicht die Herstellung gleicher Lebensverhältnisse auf der ganzen Welt, sondern lediglich die Festlegung von Rahmenvorschriften für die dringendsten globalen Probleme.

\section{Der Einwand des fehlenden Konsenses}

Ein weiterer naheliegender Einwand gegen die Möglichkeit globaler demokratischer Institutionen ist, dass es keinen internationalen Konsens über Menschenrechte und Demokratie gibt, mit denen hier argumentiert worden ist. Zwar sind die Menschenrechtspakte der Vereinten Nationen inzwischen von der großen Mehrheit der Staaten ratifiziert worden, doch gibt es nicht nur in der Praxis erhebliche Defizite, sondern auch in der Theorie Kritik an ihrer angeblich westlichen Einseitigkeit. ${ }^{159}$ Eine mehr oder weniger funktionierende Demokratie mit regelmäßigen freien Wahlen, einer Gewaltenteilung und freien Medien gibt es zur Zeit in weniger als der Hälfte aller Staaten. Wenn man also entsprechend der völkerrechtlichen Tradition davon ausgeht, dass die Legitimation einer globalen demokratischen Ordnung nur erreicht werden kann, wenn es zu einem Konsens bei ihrer Gründung kommt, ${ }^{160}$ sind die Aussichten für ihre Verwirklichung mehr als düster.

Hier geht es zunächst jedoch nur um eine „normative Zukunftsvision“. ${ }^{161}$ Ihr Hintergrund ist ein globaler Moraldiskurs, ${ }^{162}$ der schon in vielen Ländern Resonanz gefunden hat, wenn man z.B. von der Verbreitung der Schriften von Habermas ausgeht. Ungeachtet aller Kontroversen sind Demokratie und Menschenrechte normative Postulate von universalem Anspruch, sie können aber an unterschiedliche Kulturen angepasst werden. ${ }^{163}$ Auf ihrer Grundlage müssen „Modellvorstellungen für ein institutionelles Arrangement" entwickelt werden, durch das demokratische Legitimität gesichert werden kann. ${ }^{164}$

Eine internationale demokratische Organisation könnte stufenweise entstehen und attraktiv für immer mehr Staaten werden. ${ }^{165} \mathrm{Da}$ die vielfältigen Probleme bei der Ausgestaltung eines Weltparlaments sicher nicht auf einmal gelöst werden können, spricht eine pragmatische Betrachtungsweise dafür, eine solche Institution zunächst

158 Vgl. Held, Global Order (Fn. 14), S. 233: „multiple citizenships“; ähnlich Hanschmann, Homogenität (Fn. 26), S. 85 ff.: „multiple demoi“; s.a. Peters, Dual Democracy (Fn. 79), S. 297 ff.

159 Vgl. dazu z.B. Kälin/Künzli, Menschenrechtsschutz (Fn.95), S. 22 ff.; Gegenkritik auch bei Held, Demokratie (Fn. 9), S. 269 ff.

160 Held, Global Order (Fn. 14), S. 231.

161 McGrew, Grenzen (Fn. 8), S. 388.

162 Benhabib, Kosmopolitismus (Fn. 26), S. 19 (24).

163 Müller, Weltrecht (Fn. 17), S. $54 \mathrm{f}$.

164 Habermas, Rationalität (Fn. 120), S. 443.

165 Held, Global Order (Fn. 14), S. 232. 
als Angebot zu verstehen, das schrittweise Bedeutung gewinnen kann. ${ }^{166}$ Auch die Europäische Union ist zunächst nur von sechs Staaten gegründet worden, in einer Situation intensiver Konfrontation durch die Teilung Europas. Nachdem diese überwunden wurde, ist inzwischen die Mehrzahl der europäischen Staaten Mitglied geworden und der Beitrittsprozess noch keineswegs beendet. Denkbar wäre etwa, dass sich die Europäische Union Partner in anderen Kontinenten sucht, die einer solchen Institution gegenüber aufgeschlossen sind und ein Modell entwickelt, das später im Rahmen einer umfassenden Reform der Vereinten Nationen in deren Struktur integriert wird.

\section{Der Einwand der Tyrannisgefahr}

Ebenfalls schon von Kant geäußert wurde der vierte Einwand, wonach der Weltstaat zu einer globalen Tyrannis werden könnte. ${ }^{167}$ Wenn es keine konkurrierenden Staaten mehr gebe, könne sich Widerstand gegen eine Diktatur nicht mehr von außen organisieren. Insbesondere würde eine Zentralisierung der militärischen bzw. polizeilichen Gewaltmittel die Chancen, Widerstand gegen Unterdrückung zu leisten, drastisch verringern.

Dagegen ist zu betonen, dass die hier entwickelte Konzeption keinen zentralen Weltstaat anstrebt. Vielmehr soll schrittweise ein föderales System errichtet werden, das wesentlich auf dem Subsidiaritätsprinzip beruht. ${ }^{168}$ Dem Weltparlament soll nur eine begrenzte, themenspezifische internationale Rechtsetzungsbefugnis übertragen werden, die mit einer staatenunabhängigen internationalen Gerichtsbarkeit kombiniert werden muss. Auf eine globale Exekutive könnte zwar nicht vollständig verzichtet werden, wenn man das Problem der Implementationskontrolle ernst nimmt. Der Vollzug globaler Rahmennormen könnte aber wie in der EU weitgehend dezentral organisiert werden, wo ein ausdifferenzierter Verwaltungsapparat erst jetzt, nach mehreren Jahrzehnten allmählich entsteht, die Sicherheitsapparate aber weiterhin trotz der Schaffung von Kooperationsstrukturen wie Europol grundsätzlich allein in nationaler Verantwortung agieren. Auch die Polizei könnte dezentral organisiert bleiben, lediglich für Koordinationsfunktionen wäre eine globale Instanz notwendig. Wenn im Sinne des alten Ziels des ewigen Friedens das Weltrecht seine friedensstiftende Funktion verwirklichen könnte, würden militärische Kräfte ohnehin überflüssig. ${ }^{169}$

166 Möllers, Demokratie (Fn. 109), S. 106.

167 Kant, Frieden (Fn. 141), S. 225; ebenso z.B. Steiger, Weltrepublik (Fn. 13), S. 265; E. Denninger, Recht in globaler „Unordnung“, in: ders., Recht in globaler Unordnung, Berlin 2005, S. 493 (499 f.).

168 Höffe, Globalisierung (Fn. 18), S. 292 ff.; s.a. Held, Global Order (Fn. 14), S. 235 ff.; Ekardt, Demokratie (Fn. 94), S. 167; A. Niederberger, Demokratie unter Bedingungen der Weltgesellschaft?, Berlin 2009, S. 389.

169 Held, Global Order (Fn. 14), S. 276, hält eine „coercive power“ für notwendig. 


\section{F. Ausblick}

Die Diskussion über Modelle einer postnationalen Demokratie auf internationaler Ebene steht erst am Anfang. Es kann natürlich nicht erwartet werden, dass eine mehrere hundert Jahre dauernde Tradition nationalstaatlicher Institutionalisierung von heute auf morgen theoretisch und praktisch über Bord geworfen wird. Die Demokratisierung der globalen Ordnung ist ein langfristiger Prozess. ${ }^{170}$ Es ist aber an der Zeit, die Idee der Demokratie auf der Grundlage der universellen Menschenrechte zu modernisieren und von ihrer an Denkverbote grenzenden nationalstaatlichen Borniertheit zu lösen.

Transnationale Probleme erfordern handlungsfähige transnationale Institutionen. Man könnte dem hier skizzierten Modell eines Weltparlaments Fantasielosigkeit vorwerfen. Brauchen die neuen Herausforderungen globaler Demokratie nicht auch neue Strukturen? Abgesehen von den Mängeln einiger Vorschläge, die bereits angesprochen wurden (D.I.-III.), könnten auch noch andere institutionelle Ideen entwickelt werden. ${ }^{171}$ Jedoch sind die Grundstrukturen des liberalen Verfassungsstaates, nämlich eine gewählte Legislative, eine unabhängige Justiz und eine verantwortliche Exekutive, auf der Ebene der Einzelstaaten nach wie vor ohne Alternative. Ergänzungen durch direktdemokratische Elemente und verschiedene Formen der Dezentralisierung gewinnen an Bedeutung, aber eine grundlegend andere institutionelle Ordnung, die ebenfalls Demokratie garantieren kann, ist bis heute nicht bekannt. Insbesondere ist es auffällig, dass sich die EU, insbesondere durch die kontinuierliche Stärkung des Parlaments, seit der Gründung der drei Gemeinschaften eindeutig in die Richtung einer föderalen, staatsähnlichen institutionellen Struktur entwickelt hat. ${ }^{172}$ Warum sollte ein so erfolgreiches Modell nicht auch für die globale Ebene verwendet werden?

Zunächst geht es darum, die hierfür notwendige konzeptionelle Debatte zu erweitern und zu vertiefen. Welche Erkenntnisse ergeben sich aus einer vertieften verfassungsvergleichenden Analyse der demokratischen Strukturen von föderalen Systemen? Welche Ansatzpunkte liefert eine demokratische Perspektive auf den internationalen Menschenrechtsschutz? In welche Richtung entwickeln sich die internationalen Organisationen? Wie könnte ein Weltparlament ausgestaltet werden? Die Rechtswissenschaft kann zur Herausbildung einer Theorie der postnationalen Demokratie einen wichtigen Beitrag leisten.

170 Habermas, Weltgesellschaft (Fn. 14), S. 330.

171 Für eine Kombination verschiedener Ansätze Peters, Dual Democracy (Fn. 79), S. 341; ein „heterarchisches" Modell entwickelt Niederberger, Demokratie (Fn. 168), S. 405 ff.

172 Vgl. bereits T. Groß, Zwei-Kammer-Parlamente in der Europäischen Union, ZaöRV 63 (2003), S. 29 ( $50 \mathrm{ff}$.). 